\title{
Improved Holistic Analysis of Rayleigh Waves for Single- and Multi-Offset Data: Joint Inversion of Rayleigh-Wave Particle Motion and Vertical- and Radial-Component Velocity Spectra
}

\author{
Giancarlo Dal Moro, ${ }^{1}$ (D) SAyed S. R. Moustafa, ${ }^{2}$ and Nassir S. Al-Arifi ${ }^{2}$
}

\begin{abstract}
Rayleigh waves often propagate according to complex mode excitation so that the proper identification and separation of specific modes can be quite difficult or, in some cases, just impossible. Furthermore, the analysis of a single component (i.e., an inversion procedure based on just one objective function) necessarily prevents solving the problems related to the non-uniqueness of the solution. To overcome these issues and define a holistic analysis of Rayleigh waves, we implemented a procedure to acquire data that are useful to define and efficiently invert the three objective functions defined from the three following "objects": the velocity spectra of the vertical- and radialcomponents and the Rayleigh-wave particle motion (RPM) frequency-offset data. Two possible implementations are presented. In the first case we consider classical multi-offset (and multi-component) data, while in a second possible approach we exploit the data recorded by a single three-component geophone at a fixed offset from the source. Given the simple field procedures, the method could be particularly useful for the unambiguous geotechnical exploration of large areas, where more complex acquisition procedures, based on the joint acquisition of Rayleigh and Love waves, would not be economically viable. After illustrating the different kinds of data acquisition and the data processing, the results of the proposed methodology are illustrated in a case study. Finally, a series of theoretical and practical aspects are discussed to clarify some issues involved in the overall procedure (data acquisition and processing).
\end{abstract}

Key words: Surface wave dispersion, joint inversion of seismic data, Rayleigh waves, holistic analysis of surface waves, Pareto optimality, Rayleigh-wave Particle Motion (RPM) curve, Rayleigh-wave Prograde motion.

1 Institute of Rock Structure and Mechanics, Academy of Sciences of the Czech Republic, V Holešovičkách 94/41, 18209 Prague 8, Czech Republic. E-mail: dalmoro@irsm.cas.cz

2 Geology and Geophysics Department, Faculty of Sciences, King Saud University, King Saud Street, Riyadh 11451, Saudi Arabia.

\section{Introduction}

The exploitation of surface-wave propagation for the determination of the vertical shear-wave velocity $\left(V_{\mathrm{S}}\right)$ profile is nowadays routinely adopted for a number of seismological and geotechnical applications (e.g., Poggi and Fäh 2010; Luo et al. 2011; O'Connell and Turner 2011; Boxberger et al. 2011; Zhang et al. 2017).

A number of active and passive techniques aimed at retrieving the dispersive properties of the investigated site have been proposed (for an overview, see Dal Moro 2014; Foti et al. 2014) but we should highlight that the way we then invert the obtained dispersive properties is a different issue.

Surface-wave analysis is in fact typically accomplished in two steps:

1. determination of the dispersive properties of the site;

2. their inversion (aimed at determining the subsurface $V_{\mathrm{S}}$ model).

The multi-channel analysis of surface waves (MASW) is a very well known acronym typically used to indicate the classical approach to determine the dispersive properties from multi-channel (multioffset) active data. A series of equally spaced geophones is deployed and the acquired seismic traces are used to define the phase velocity spectrum (Xia et al. 1999; Dal Moro et al. 2003).

Typically, only the vertical-component of Rayleigh waves is considered and the obtained velocity spectrum (which represents the dispersive properties of the site) is interpreted in terms of modal curves 
which are then inverted (Xia et al. 1999; Ryden et al. 2003).

It must be emphasized that such approach (the interpretation of the modal dispersion curves of the vertical-component of Rayleigh waves, their picking and final inversion) is not the only possible and, actually, it can be highly problematic because it involves a personal (i.e., subjective) interpretation of a single velocity spectrum (see Zhang and Chan 2003; Dal Moro 2014; Dal Moro et al. 2015a, b, c).

With the aim of illustrating how complex and counterintuitive a phase velocity spectrum can actually be, in Fig. 1 we present and comment a synthetic dataset computed according to Carcione (1992). The
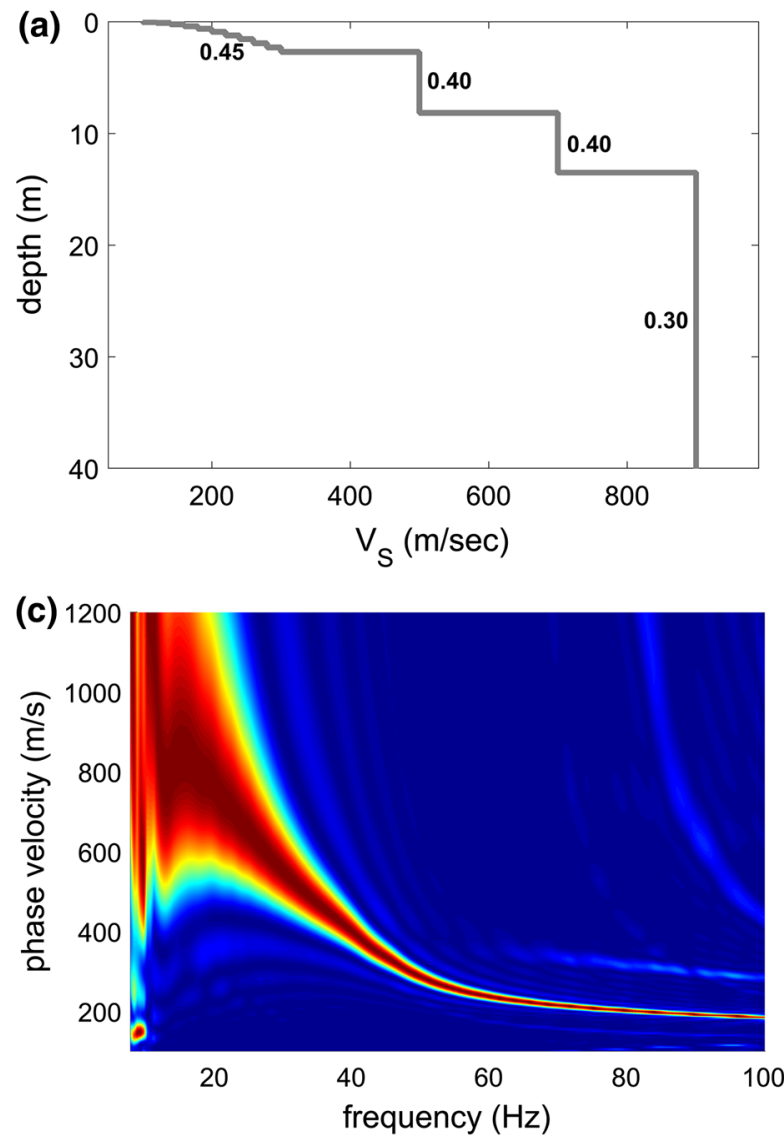

computed phase velocity spectrum reported in Fig. 1c is apparently continuous and seemingly simple (no jump or weird features). In spite of this, once we plot the theoretical dispersion curves of the first two modes (Fig. 1d), we realize that the signal that dominates the velocity spectrum is actually the combination of the fundamental mode for frequencies higher than $40 \mathrm{~Hz}$ and of the first higher mode for lower frequencies.

Because the signal in the velocity spectrum is continuous (Fig. 1c), it is clear that in case this kind of dataset would be interpreted in terms of modal dispersion curves, it would be inevitably misinterpreted and, consequently, an erroneous subsurface
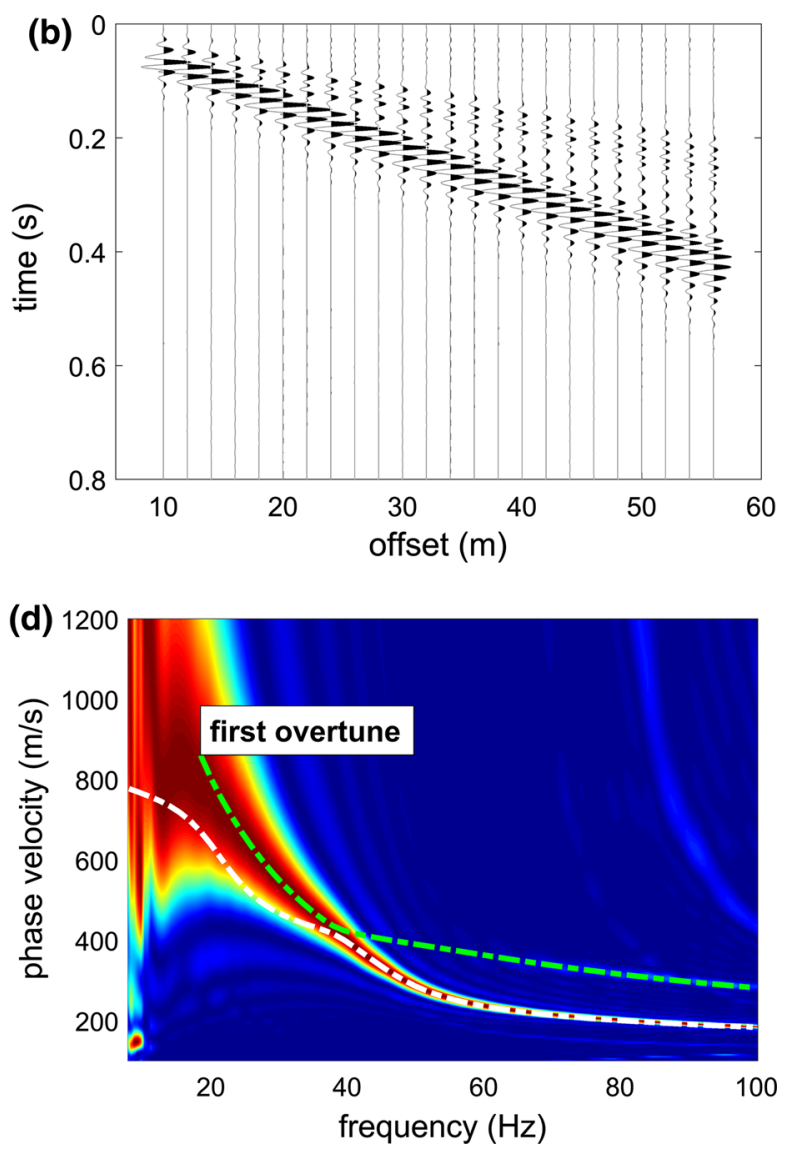

Figure 1

Complex (counterintuitive) phase velocity spectrum for a synthetic dataset (MASW data): a subsurface model (the numbers are the adopted Poisson's values); b synthetic seismic traces of the vertical-component computed according to Carcione (1992); c phase velocity spectrum of the computed synthetic traces; $\mathbf{d}$ phase velocity spectrum and theoretical modal dispersion curves of the first two modes (the white and green lines indicate the fundamental and first higher mode, respectively). Because the signal in the velocity spectrum is continuous (see plot c), it is impossible to separate the two modes. Consequently, any kind of analysis based on the identification and inversion of the modal dispersion curves will necessarily fail 
model would be obtained. Since the velocity spectrum would be likely interpreted as expression of the fundamental mode only, the obtained $V_{\mathrm{S}}$ values would be clearly overestimated.

In Dal Moro et al. (2015a, b, 2016), we introduced a series of procedures aimed at the joint analysis of multi-component data with the final goal of obtaining a subsurface model free from significant ambiguities both because we analyze multi-component data, both because we do not consider an approach based on the analysis of picked (i.e., subjectively interpreted) modal dispersion curves.

One of the goals of the present work is to further improve the analysis of Rayleigh waves and, therefore, give the MASW acronym a more comprehensive meaning.

In fact, several points should be addressed when dealing with surface-wave analysis:

1. How many and what kind of components are considered? Only the vertical component? Only the vertical and radial components of Rayleigh waves? Are Love waves analyzed?

2. How are the data analyzed and inverted? Do we deal with interpreted modal dispersion curves? According to the effective dispersion curve approach (Lai and Rix 1998; 2002)? Is the entire frequency-velocity matrix considered (Dal Moro et al. 2014; Dal Moro 2014)?

3. Do we only consider the velocities or any other ancillary "object" useful to further characterize the surface-wave propagation and consequently better constrain the inversion process?

Although the importance of Love waves was already pointed out in Safani et al. (2005), Dal Moro and Ferigo (2011), Xia et al. (2012), Dal Moro (2014), and Dal Moro et al. (2015c), for the present work we focus on Rayleigh waves only.

While working on the practical aspects to face during the exploration of large areas (Dal Moro and Keller 2015), we realized that, when dealing with several hundreds or thousands of shot points, the joint acquisition of Rayleigh and Love waves might be quite cumbersome and, consequently, expensive. In fact, the joint acquisition of Rayleigh and Love waves requires the use of two different sources and, for the shear source used to generate Love waves, obtaining a good coupling can be sometimes problematic. Although these facts can be easily handled while dealing with few shots, when the amount of shot points becomes large, the overall acquisition procedures can become hard to manage.

We then focused on the opportunities highlighted in some recent studies regarding the holistic analysis of Rayleigh waves (Dal Moro et al. 2015a, b, 2017).

By holistic we refer to the analysis of various attributes that, altogether, can fully describe the Rayleigh-wave propagation, so not merely the phase or group velocities of one or two components.

In the present work we consider the Rayleigh waves acquired according to active procedures, but analyze their propagation both from the point of view of the velocities along the vertical $(Z)$ and radial $(R)$ components (in general terms, the $Z$ and $R$ velocity spectra are different), both considering the actual particle motion summarized by the Rayleighwave particle motion (RPM) curves.

Such a new "object" (the RPM curve) aims to describe the actual particle motion due to the Rayleigh-wave propagation and was introduced in Dal Moro et al. (2017). Although it is often stated that Rayleigh waves propagate according to a retrograde motion, it was observed that prograde motion is possible (Tanimoto and Rivera 2005; Malischewsky et al. 2008) and actually much more common than usually believed (Dal Moro et al. 2017).

In the present work, the RPM data are jointly inverted with the velocity spectra of both the vertical $(Z)$ and radial $(R)$ components of Rayleigh waves, here analyzed according to the Full Velocity Spectrum (FVS) approach (Dal Moro 2014; Dal Moro et al. 2014, 2015c), thus not considering the classical modal dispersion curves.

The goal is to understand and properly exploit complex datasets and define a robust subsurface model that does not significantly suffer from data misinterpretation and ambiguities related to the nonuniqueness of the solution.

It is important to point out that the RPM curves quantify the retrograde or prograde motion and not the Rayleigh-wave ellipticity as described for instance in Hobiger et al. (2009) for passive data and Dal Moro et al. (2015a, b, 2016) for active data. The RPM data describe the verse (retrograde, prograde, or 
a mixture of the two) of the motion, while the ellipticity quantifies the shape (more or less elliptical) of the Rayleigh-wave circular motion.

In the present paper, the velocity spectra of the Rayleigh-wave vertical- and radial-components are jointly analyzed with the RPM data according to two possible approaches:

1. multi-offset data that can be acquired by a classical multi-channel seismograph;

2. single-offset data acquired by a single threecomponent (3C) geophone deployed at a certain distance (offset) from the source.

To avoid exceedingly wordy expressions, we refer to the two approaches using the following expressions and related acronyms: multi-offset RPM holistic (MO-RPM-HS) analysis when dealing with multi-offset data and single-offset RPM holistic (SORPM-HS) analysis for single-offset data.

In the Sect. 2, we first describe the various and different acquisition procedures that can be adopted and summarize some fundamental points regarding the data analysis.

Then, in the Sect. 3, we describe the way the three considered objects are handled and inverted.

The results obtained by means of the two possible approaches (MO-RPM-HS and SO-RPM-HS) are illustrated by considering a non-trivial field dataset.

A further dataset, largely dominated by prograde motion, is then analyzed and discussed to highlight the importance of the proposed joint approach.

We eventually summarize the main advantages of the proposed method, also briefly presenting a 2D section obtained by means of the proposed holistic approach.

\section{Data Acquisition}

Since the methodology considered in the present paper can be used for both single- and multi-offset data, in the next three sub-sections we summarize three different approaches that can be adopted to collect the multi-component data required for the holistic analyses described later on.

\subsection{Acquisition of Single-Offset Multi-Component Data (SO-RPM-HS Approach)}

The Rayleigh-wave analysis accomplished with the classical multiple-filter analysis (MFA) method (Dziewonsky et al. 1969) was recently improved by considering a multi-component inversion based on three objective functions (Dal Moro et al. 2015a, b, 2016): the group-velocity spectra of the radial and vertical-components and the radial-tovertical spectral ratio (RVSR).

The key point of this approach (holistic analysis of surface waves; HS) is that the data acquisition is performed by a single $3 \mathrm{C}$ geophone positioned at a certain distance (offset) from the source (Fig. 2). Clearly, the maximum penetration depth depends on the lowest frequency that can be soundly analyzed, which depends on the combination of several facts: the characteristics of the adopted source, the attenuation induced by the local sediments and the adopted offset (the larger the distance between the source and the receiver, the deeper the potential penetration depth). For a wider discussion about these mutually related aspects see for instance Luo et al. (2011) and Foti et al. (2014).

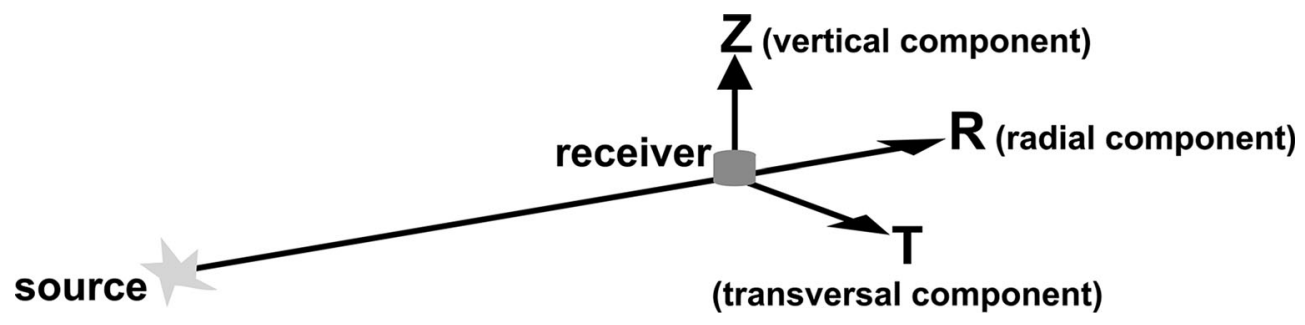

Figure 2

Single-offset multi-component data acquisition: a single 3-component geophone is set at a defined distance (offset) from the source. For the implementation of the method considered in the present paper, we only consider the $Z$ and $R$ components 
The recorded data can also be used to compute the RPM frequency curve (Dal Moro et al. 2017), which will be here jointly analyzed with the velocity spectra of the $\mathrm{Z}$ and $\mathrm{R}$ components.

It is useful to underline the meaning of two expressions that might be otherwise confused. In spite of the fact that in this latter case (Fig. 2) we are dealing with only two or three seismic traces, the acquisition is still multi-channel since it requires more than one acquisition channel. In other words, the expression multi-channel does not necessarily refer to the use of a series of single-component geophones set at different offsets (conventional MASW approach): the acquisition setting represented in Fig. 2 is multi-channel and single-offset since it allows the acquisition of multi-component data $(Z$, $R$ and $T$ components) referred to a single offset.

On the other side, if we consider 24 vertical geophones set at increasing distances from the source (standard MASW acquisition setting providing single-component data for several offsets), we deal with a multi-offset and multi-channel setting that allows the acquisition of just one component (in this case the vertical one).

\subsection{Acquisition of Multi-Offset Multi-Component Data (MO-RPM-HS Approach)}

Multi-offset and multi-component data can be easily and simultaneously acquired using a common and classical 24-channel seismograph and the acquisition scheme reported in Fig. 3 (please notice that for most of the near-surface applications, 12 traces are more than sufficient for surface-wave dispersion analysis_Dal Moro et al. 2003; Dal Moro 2014).

However, the $Z$ and $R$ components do not have to be acquired simultaneously, as in the scheme reported in Fig. 3. In case we want to deal with a 24-trace dataset and have a 24-channel seismograph, it is possible to acquire first the 24 vertical traces and then the 24 radial traces, by simply replacing the geophones.

Needless to say that an acquisition system equipped with $3 \mathrm{C}$ sensors would allow the simultaneous and quick acquisition of all the components.

From the practical point of view, it is important to point out that the vertical and horizontal geophones do not need to have exactly the same response curve because the RPM curve does not depend on the amplitudes of the two traces-details are provided in Sect. 3.2. This is quite relevant because the two sets of vertical and horizontal geophones might come from different manufacturers and have different response curves.

In contrast, the computation of the RVSR (the curve that describes the Rayleigh-wave ellipticity in case of active data used for instance in Dal Moro et al. 2015a, b, 2016) requires calibrated geophones with the same response curve.

\section{Rayleigh Waves}

Simultaneous Joint Acquisition of the Vertical (Z) and Radial (R) Components

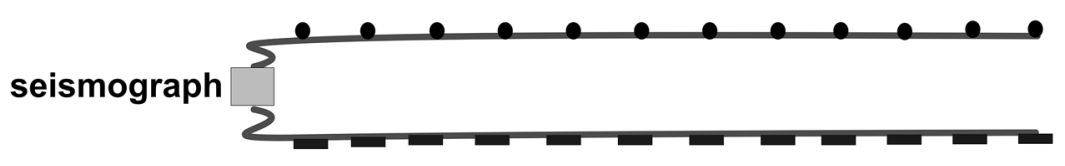

$\bullet \mathrm{Z}$ (vertical) geophone $\quad-\mathrm{R}$ (horizontal/radial) geophone

Figure 3

Example of multi-offset joint simultaneous acquisition of the $Z$ and $R$ components (multi-component MASW approach) 


\subsection{Multi-Offset and Multi-Component Acquisition via Multi-channel Simulation with One Receiver (MSOR)}

Multi-offset and multi-component data can also be recorded using a single $3 \mathrm{C}$ geophone and moving the source to different offsets, which is sometimes called multi-channel simulation with one receiver (MSOR; Ryden et al. 2003).

This acquisition technique can provide multioffset and multi-component data suitable for our analyses using affordable equipment.

Compared to Ryden et al. (2003), in our case, we would use not a single-component sensor, but a $3 \mathrm{C}$ geophone that allows the simultaneous acquisition of both the vertical- and radial-components for each offset (in the present paper we do not consider the transversal component); hence, the expression "multi-offset simulation with one $3 \mathrm{C}$ receiver" would be actually more precise.

The main problem in applying the MSOR approach, is that the field procedures are necessarily relatively heavy (usually the geophone remains in one place and the source moves). To reduce the physical effort related to the hammer work and source re-location, the stack adopted for each singleoffset is then often small and, consequently, the signal-to-noise ratio risks to be poor. This can be significant in some cases, especially for the radial
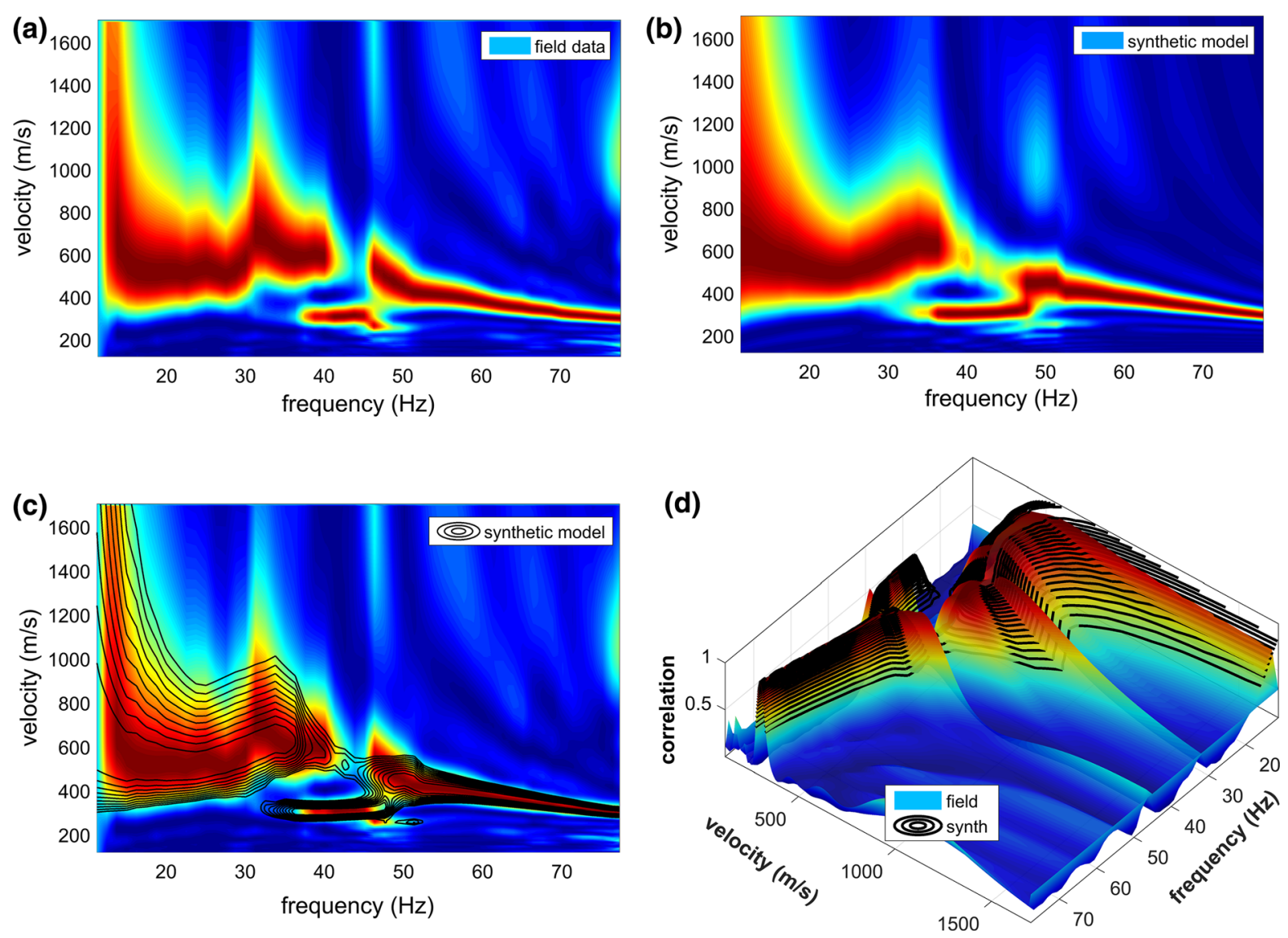

Figure 4

Concise representation of the Full Velocity Spectrum (FVS) analysis: a phase velocity spectrum of a field dataset; b phase velocity spectrum of the synthetic model identified by means of the FVS optimization procedure presented in Dal Moro et al. (2014, 2016) and Dal Moro (2014); c compact representation of the two previous velocity spectra (the background colors represent the velocity spectrum of the field data, while the overlaying black contour lines pertain to the synthetic data); d same data as in the previous plot but from a different (3D) perspective 
component, which is often more sensitive to heterogeneities between the source and the receivers (e.g., Rodríguez-Castellanos et al. 2006).

\section{The Three Objects and Their Joint Inversion}

As briefly stated in the introductory paragraph, the proposed method is based on the joint inversion of three "objects" that, altogether, holistically describe the way Rayleigh waves propagate:

1. the velocity spectrum of the vertical component;

2. the velocity spectrum of the radial component;

3. the RPM data describing the actual particle motion.

The velocity spectra of the radial and vertical components are analyzed and inverted according to the FVS approach. As shown in the Sect. 3.1, this approach (widely described in Dal Moro et al. 2014 and Dal Moro 2014) allows the analysis of the velocity spectra without their interpretation in terms of modal dispersion curves.

The RPM data are represented by a single RPM frequency curve when we consider the SO-RPM-HS approach (one single offset), or by a RPM frequencyoffset surface in case of multi-offset data (MO-RPMHS).

Some fundamental aspects regarding the FVS approach and RPM data are described in the following three sub-sections, together with a brief description of the adopted multi-objective inversion scheme.

\subsection{Full Velocity Spectrum (FVS) Analysis}

Dispersion analysis is commonly performed while considering interpreted modal dispersion curves. By the way, extracting the modal dispersion curves from a field velocity spectrum is not always easy or feasible since it can be hard to define and separate different modes (see Fig. 1 and related text) and, in
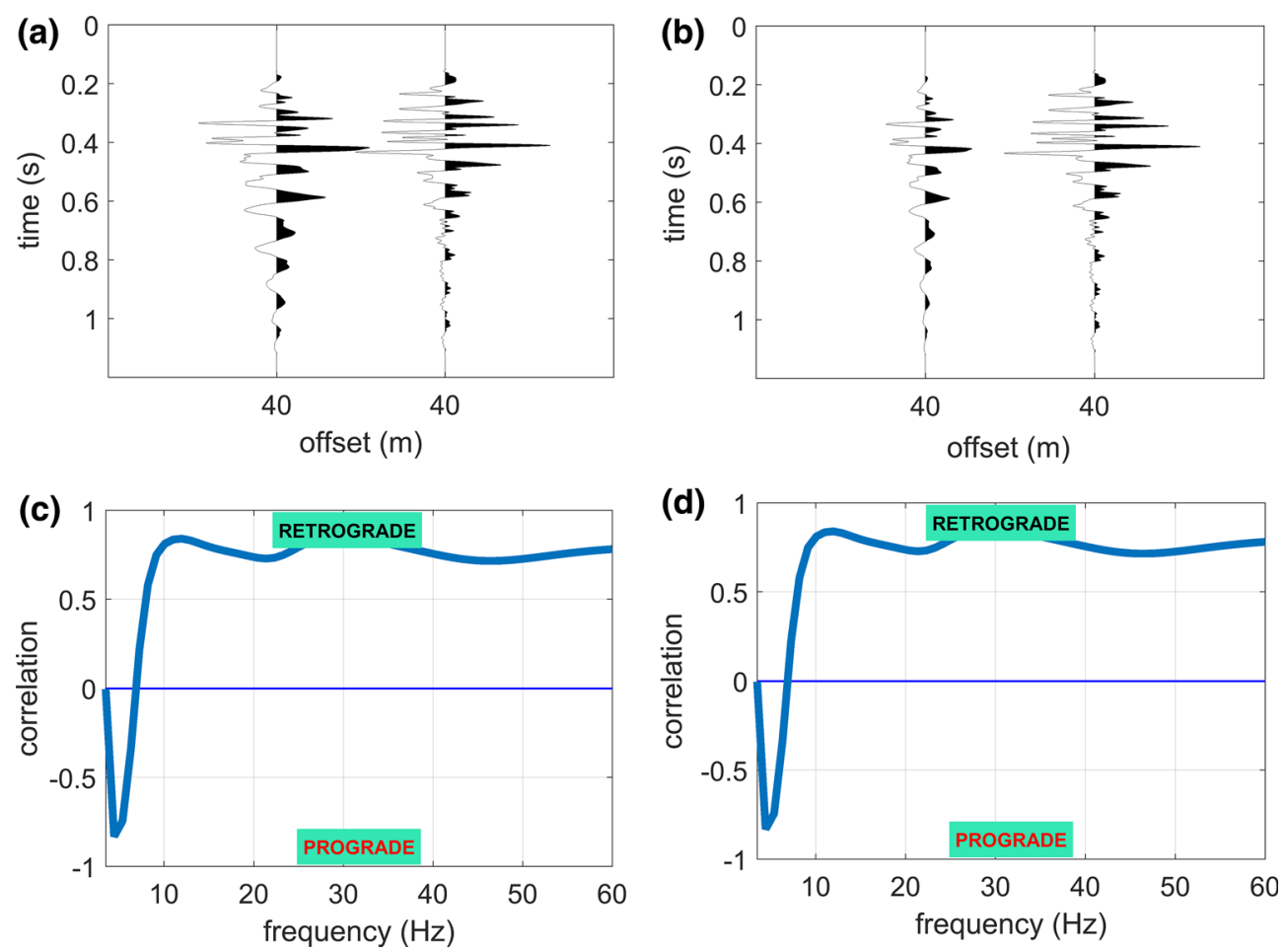

Figure 5

Computation of the Rayleigh-wave particle motion (RPM) frequency curve: a original vertical $(Z)$ and radial $(R)$ field traces (offset $40 \mathrm{~m})$; b manipulated traces (the $Z$ trace is divided by 2); c, $\mathbf{d}$ report the RPM curves of the original and manipulated data, respectively 
any case, the picking of a modal dispersion curve is a subjective exercise. In other words, the inversion of a picked dispersion curve is not the inversion of an objective quantity but of an interpreted (i.e., subjective) curve.

To try to overcome these issues, through the FVS approach we invert the velocity spectrum as a whole without the need to name the modes in it. Actually, the first two objects we consider in the implemented methodology are the velocity spectra of both the radial and vertical components of Rayleigh waves which, in general terms, are different, so provide complementary information.

The FVS inversion is accomplished through the following scheme:

1. computation of the synthetic seismic traces of the considered components for a tentative model;

2. computation of the velocity spectra of the obtained synthetic traces (via phase shift in case of phase velocities or MFA in case of group velocities);

3. computation of the misfit between the velocity spectra of the field and synthetic data.

These three steps are implemented within an optimization scheme that minimizes the misfit and eventually provides the subsurface model associated with a velocity spectrum as similar as possible to the velocity spectrum of the field data. In this way we deal with the entire velocity spectrum (i.e., the entire frequency-velocity matrix) and not with a picked dispersion curve (i.e., an interpreted frequency-velocity curve). Further details and a series of case studies are presented in Dal Moro 2014 and Dal Moro et al. 2014, 2015a, c, 2016).

An example of single-component FVS inversion is reported in Fig. 4. The first plot (Fig. 4a) reports the phase velocity spectrum of a field dataset, while Fig. $4 \mathrm{~b}$ shows the velocity spectrum of the synthetic dataset obtained by following the abovereported optimization scheme. To summarize both the velocity spectra in a single plot, Fig. 4c displays both the field (background colors) and synthetic (overlaying black contour lines) spectra (the 3D plot in Fig. 4d contains the same data from a different perspective).
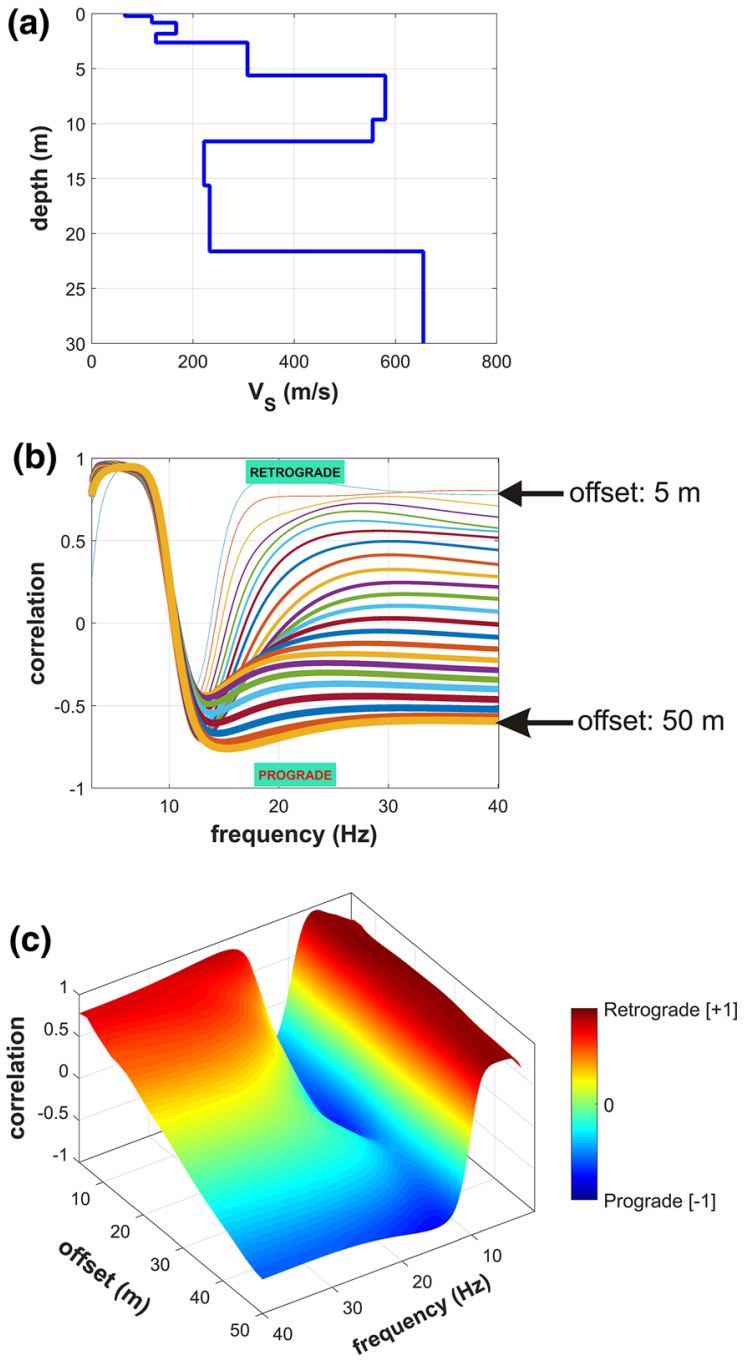

Figure 6

Rayleigh-wave particle motion (RPM) data for a synthetic multioffset dataset: a $V_{\mathrm{S}}$ subsurface model (Poisson values fixed to 0.33 for all layers); b RPM frequency curves for 24 different offsets ranging from 5 to $50 \mathrm{~m}$ (the thicker the line, the larger the offset); c their representation as RPM frequency-offset surface

\subsection{The RPM Frequency Curve and its Basic Properties}

The RPM frequency curve is represented by the correlation coefficients between the radial $(R)$ component and the Hilbert transform of the vertical $(Z)$ trace computed after filtering the data with a series of narrow Gaussian filters centered at each considered frequency (Dal Moro et al. 2017). We then obtain a curve with values ranging from -1 
(pure prograde motion) to +1 (pure retrograde motion).

Such a curve summarizes the actual motion of a particle induced by Rayleigh waves and, since its

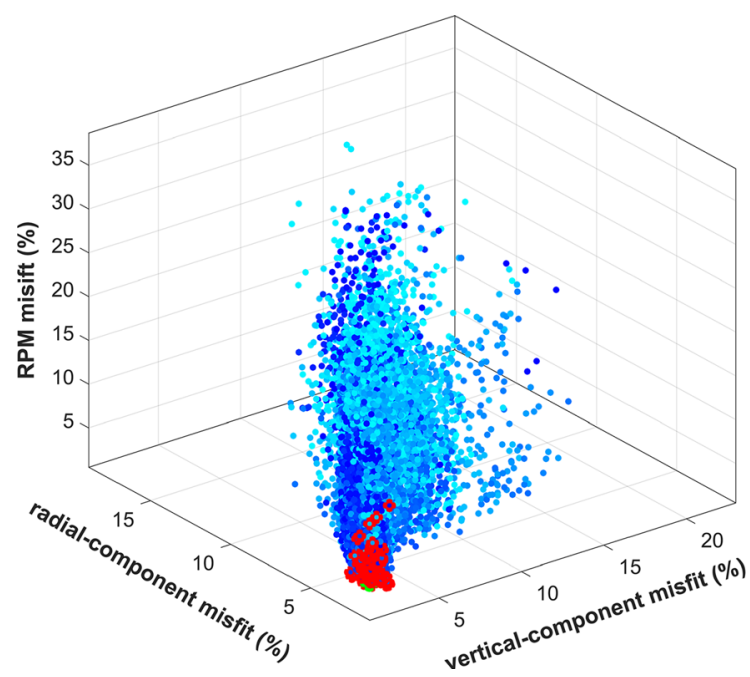

Figure 7

Multi-objective evolutionary algorithm (MOEA) optimization: misfit values of the three objective functions considered for the present study (velocity spectra of the vertical and radial components and RPM data). Each point represents a model evaluated during the optimization procedure. The red circles indicate the final Pareto optimal models actual trend depends on the subsurface conditions, it can be exploited to further constrain the subsurface model itself.

Rayleigh-wave motion is in fact frequency dependant (low frequencies are influenced by the characteristics of the deep layers, while the high frequencies depend on the shallow materials).

In this respect, compared to the polarity analysis presented in Gribler et al. (2016), the RPM frequency curve benefits in particular from two important features:

1. because it is defined frequency by frequency (and not as a mean value over a wide frequency range), it is significantly more accurate and meaningful;

2. since it is based on the computation of the correlation coefficient, it can be determined even if the vertical and horizontal geophones used to acquire the data do not have the same response curve.

The first point is quite simple and does not require much explanation; the average polarity considered by Gribler et al. (2016) is necessarily a rough value with a relatively limited meaning because the actual Rayleigh-wave motion is a function of the frequency (and offset).

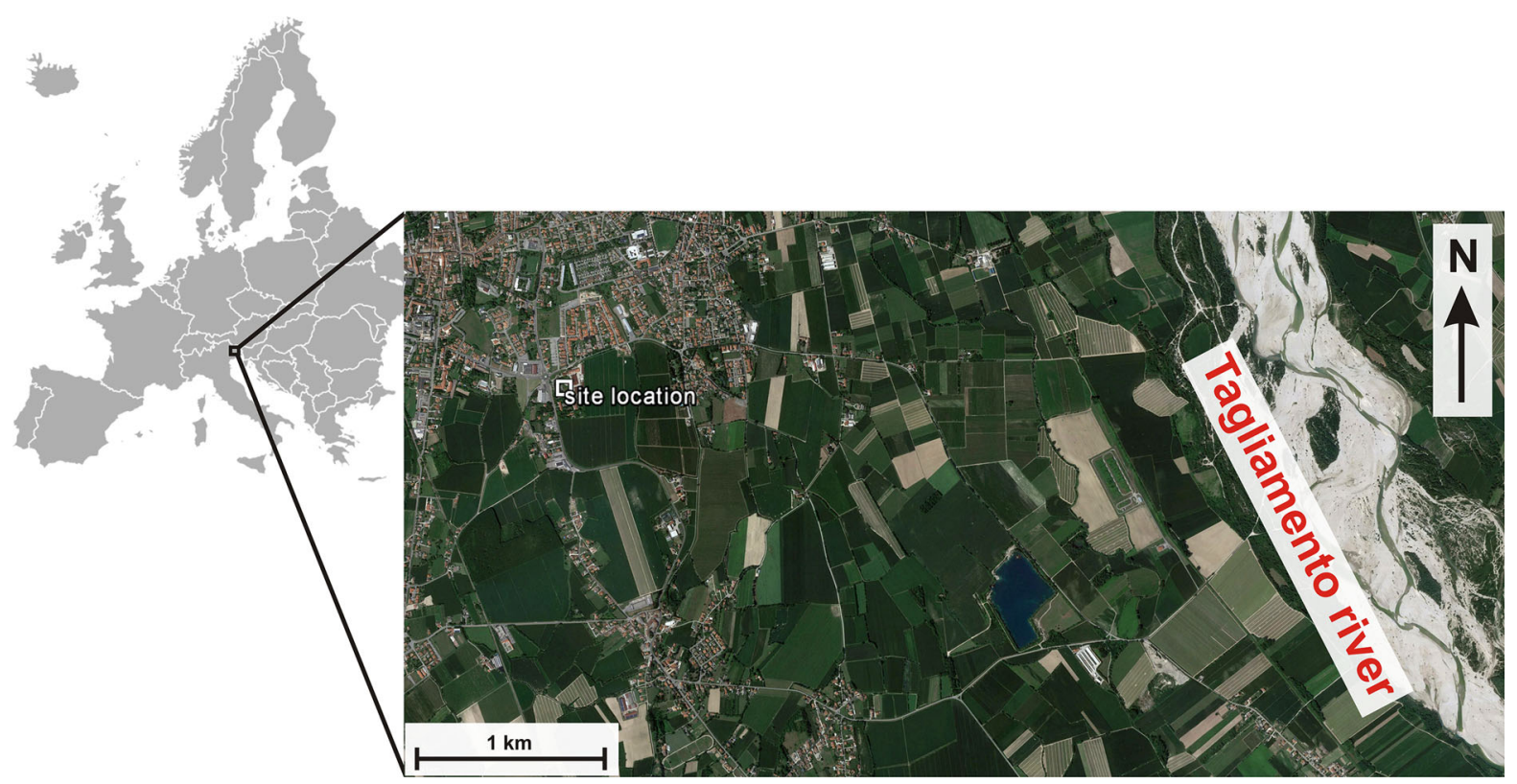

Figure 8

Location of the study site (Northeast Italy). The gravel bed of the Tagliamento River is clearly visible on the right 
Table 1

Acquisition parameters for the multi-offset and multi-component data accomplished via MSOR (Ryden et al. 2003)

Minimum offset

Station spacing

Number of stations

Record length

Sampling frequency

Stack

On the other side, because of its practical consequences, it is probably useful to clearly illustrate the second property of the RPM frequency curve.

The data reported in Fig. 5 are used to provide such evidence. The $\mathrm{Z}$ and $\mathrm{R}$ field traces reported in Fig. 5a are used to compute the RPM frequency curve presented in Fig. 5c. We then divided the $\mathrm{Z}$ trace by two (Fig. 5b) and re-computed the RPM frequency curve (Fig. 5d). The two obtained RPM curves are clearly identical and this provides the evidence that, in order to compute the RPM curve, it is not necessary to use geophones with the same response curve.

On the other hand, the method presented in Gribler et al. (2016) is based on the analysis of the amplitudes; therefore; it requires analyzing data acquired through vertical and horizontal geophones with the same response curve.

In contrast, the RPM frequency curves are defined by simple correlation coefficients and, consequently, do not depend on the amplitude of the two traces but only on their synchronization (frequency by frequency).

Because the RPM frequency curve is computed for a specific offset, in case of multi-offset data (Fig. 3) we can define a RPM frequency-offset surface. Figure 6 reports a multi-offset synthetic case for a non-trivial subsurface model with a significant low-velocity area between about 12 and $21 \mathrm{~m}$ (a)

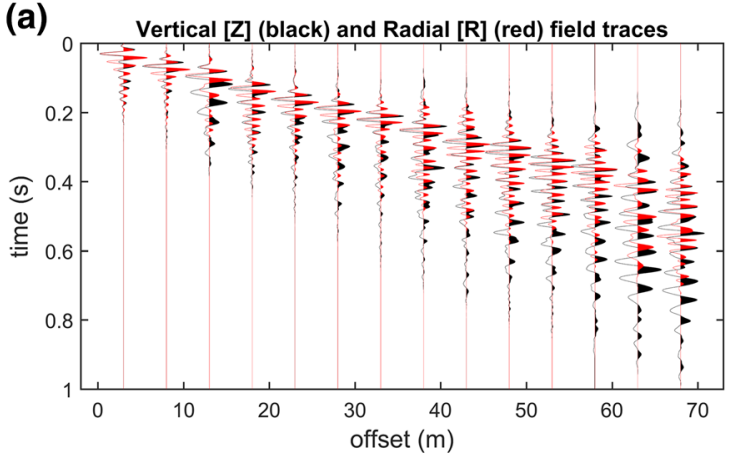

(c)

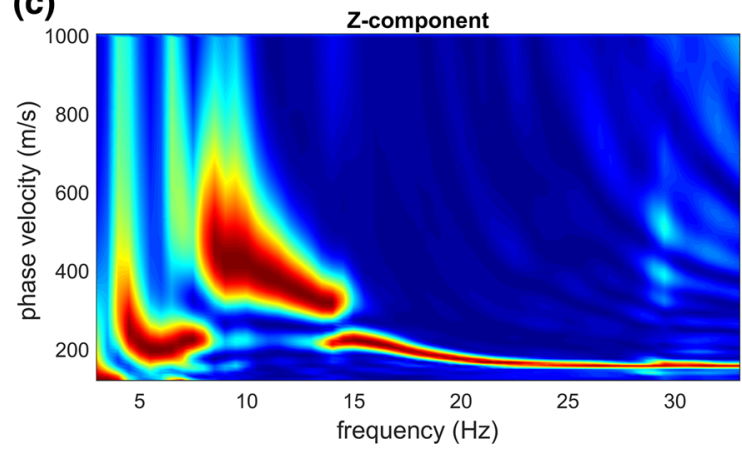

(b)

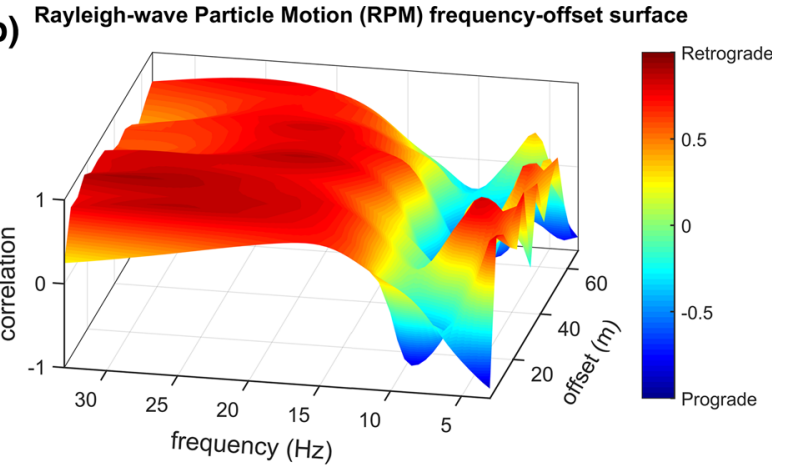

(d)

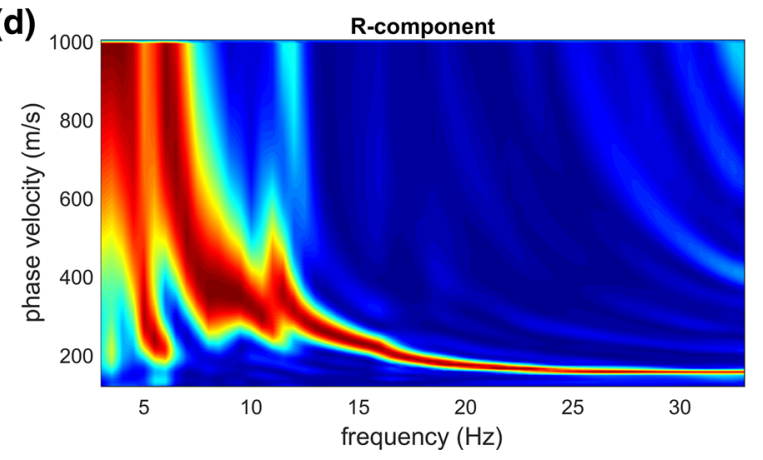

Figure 9

Case study. Field data acquired via MSOR and the three objects considered for the MO-RPM-HS approach: a vertical (Z) and radial (R) traces; b Rayleigh-wave particle motion (RPM) frequency-offset surface; c phase velocity spectrum of the vertical component; d phase velocity spectrum of the radial-component 
(Fig. 6a). The synthetic $\mathrm{Z}$ and $\mathrm{R}$ traces were created following Herrmann (2013) and from these we then computed the RPM frequency curves for each considered offset.

In Fig. $6 b$ we report the single RPM frequency curves for several offsets, while in Fig. 6c is reported the RPM frequency-offset surface which describes the RPM as a function of both the frequency and offset. As expected, the way the different modes unfold and determine the actual RPM varies both as a function of the frequency and offset.

As shown in the case studies reported in Dal Moro et al. (2017), we must emphasize that prograde motion occurs much more frequently than traditionally hypothesized, even for very simple subsurface conditions. Abrupt shear-wave impedance variations or/and low-velocity channels are often responsible for prograde motion, but even very simple subsurface conditions can actually generate prograde motion, which is actually determined by the combination of several subsurface features that cannot be always easily schematized.

\subsection{Joint Inversion via Multi-Objective Evolutionary Algorithm (MOEA) Optimization}

The joint inversion of the three considered objects (the RPM data and the velocity spectra of the vertical and radial components) is performed according to the multi-objective evolutionary algorithm (MOEA) optimization scheme described in Dal Moro and Pipan (2007) and Dal Moro et al. (2016), thus without any normalization of the misfit values and while keeping them separate.

Figure 7 shows an example of a three-objective space: each point represents a subsurface model (defined by its three misfits) and the red circles highlight the final Pareto front models (Sawaragi et al. 1985; Van Veldhuizen and Lamont 1998; Zitzler and Thiele 1999). (a)

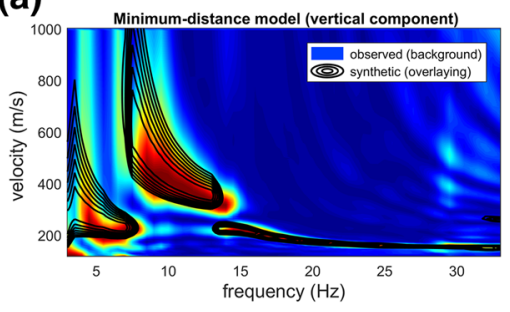

(d)

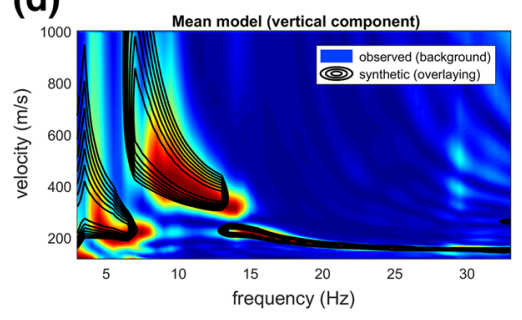

(b)

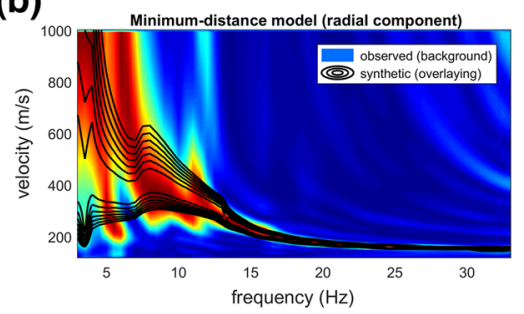

(e)

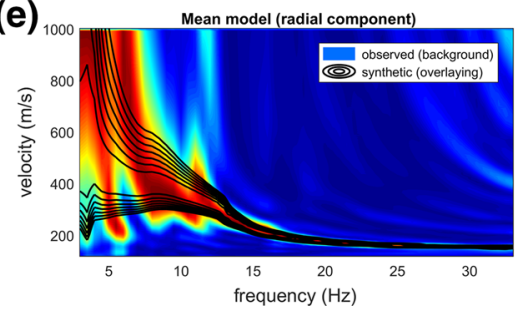

(c)

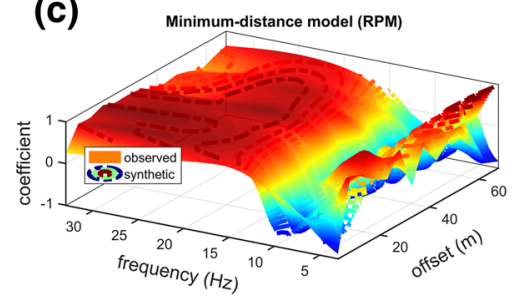

(f)

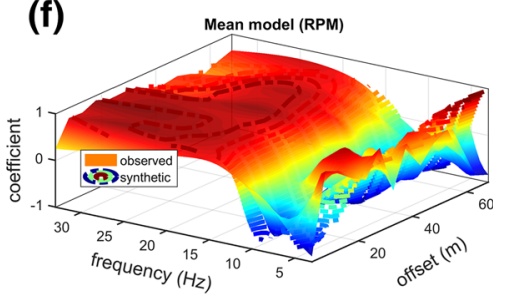

Figure 10

Case study (MO-RPM-HS approach). The two most important models obtained from the joint inversion of the multi-offset data (see also Fig. 11). Upper plots refer to the minimum-distance model: a vertical-component phase velocity spectra; b radial-component phase velocity spectra; c RPM frequency-offset surfaces. Lower plots refer to the mean model (computed by considering all the Pareto optimal models): d vertical-component phase velocity spectra; e radial-component phase velocity spectra; f RPM frequency-offset surfaces. For the velocity spectra, the colors in the background pertain to the field data, while the overlaying black contour lines represent the synthetic data of the identified models. For the RPM data, the synthetic surface is reported by dashed contour lines with the same color scale as the field data (since the agreement between the field and synthetic data is extremely good, the two surfaces are visually hardly separable) 
It is important to underline that a joint inversion is necessarily a sort of compromise. While it is simple to find a "perfect" match for a single objective function (e.g., the phase velocity spectrum or dispersion curve of the vertical-component of the Rayleigh waves), when dealing with two or more objective functions it is usually impossible to find a model that perfectly matches all the considered objects. In fact, data always and necessarily contain some noise (both coherent and incoherent) that can reflect differently on the considered components and objective functions. Rodríguez-Castellanos et al. (2006) showed for instance that, with respect to the vertical component, the radial component of Rayleigh waves is more affected by possible cracks in the medium.

A dipping horizon, the scattering created by buried boulders or discontinuities and, in general, any sort of possible anisotropy or heterogeneity can influence the various components differently.

This means that when we perform a joint inversion we cannot expect a perfect match for all the considered objective functions.

Incidentally, a quantitative way to evaluate the consistency of a joint inversion performed via MOEA was presented in Dal Moro and Pipan (2007), Dal Moro and Ferigo (2011) and Dal Moro (2014) and is based on the analysis of the symmetry of the final Pareto optimal models.

From a practical point of view, the overall MOEA procedure is accomplished through the following steps:

1. computation of the RPM curve(s) and velocity spectra of the $Z$ and $R$ components of the field data;

2. definition of a reasonable search space;

3. creation of a series of initial subsurface models (randomly defined within the search space) and computation of the synthetic traces of the $Z$ and $R$ components;

4. computation of the velocity spectra and RPM curves of the computed synthetic traces;

5. computation of the three misfits (for the three considered "objects");

6. optimization of the model (MOEA scheme).

The two most important models eventually obtained are the mean model computed by considering all the Pareto optimal models and the model (i.e., the point—see Fig. 7) having the minimum geometrical distance from the utopia point of the multi-objective space (for details see Dal Moro and Pipan, 2007 and Dal Moro et al. 2015c). In the present paper, such solution is defined as the minimum-distance model.

\section{Case Study}

A multi-offset and multi-component dataset was acquired in north-eastern Italy (Fig. 8) in a reclamation area dominated by coarse sediments (gravel) with occasional sandy layers.

Data acquisition was performed using a single 3C geophone and moving the source to different offsets

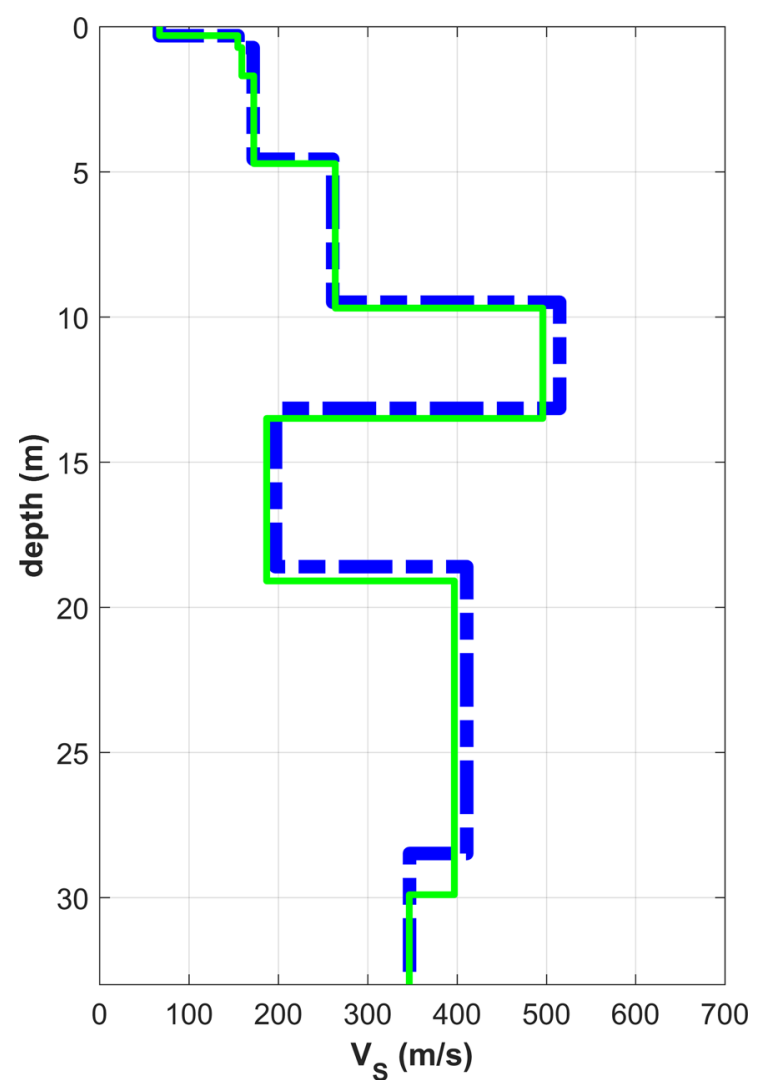

Figure 11

Case study (MO-RPM-HS approach): identified $V_{\mathrm{S}}$ models. The dashed blue line indicates the mean model (computed considering all the Pareto optimal models), while the continuous green line represents the minimum-distance model 

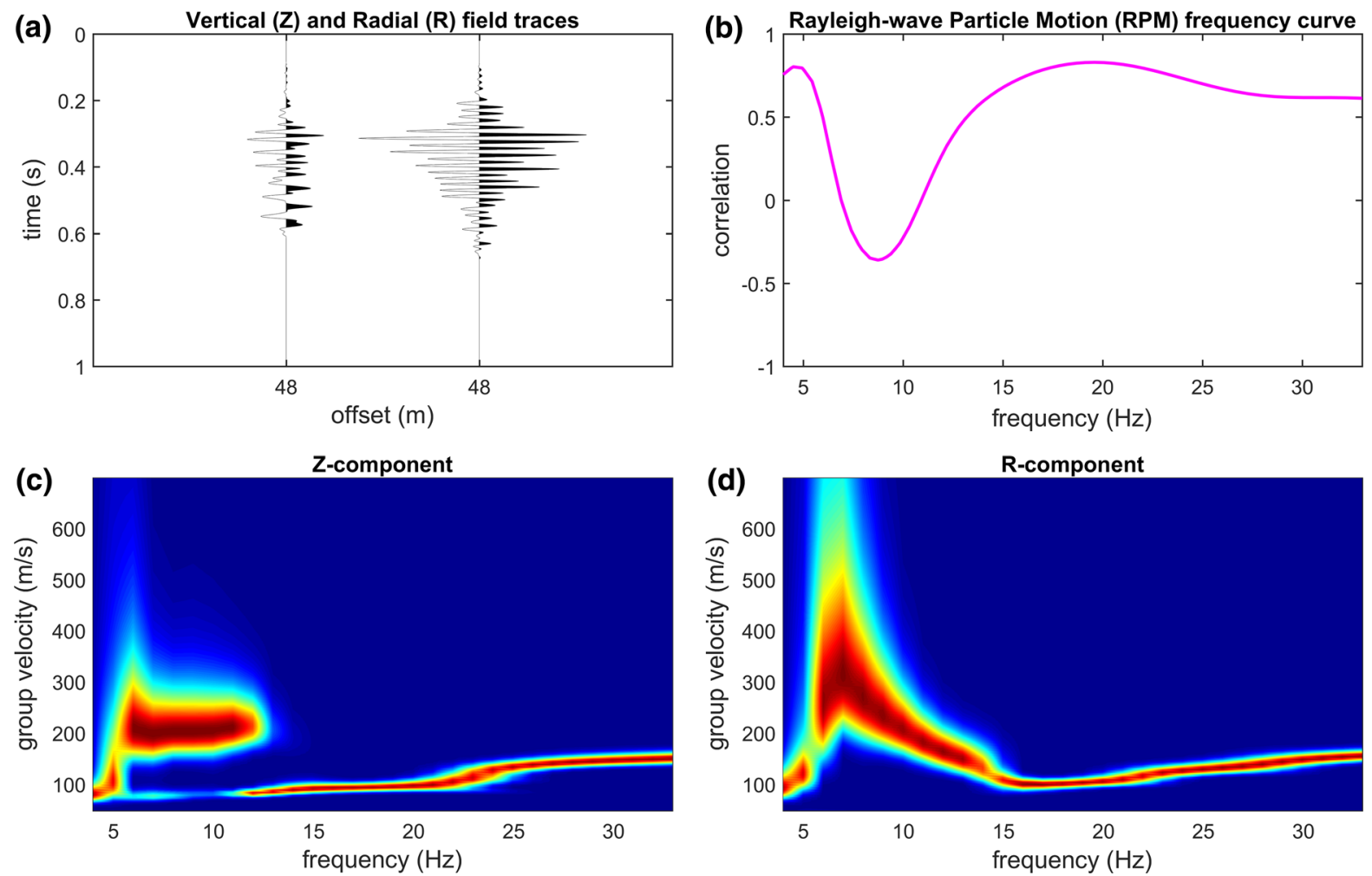

Figure 12

Case study (SO-RPM-HS approach): a $Z$ and $R$ traces (offset $48 \mathrm{~m}$ ); b RPM frequency curve; $\mathbf{c}$ group-velocity spectrum of the vertical $(Z)$ component; $\mathbf{d}$ group-velocity spectrum of the radial $(R)$ component
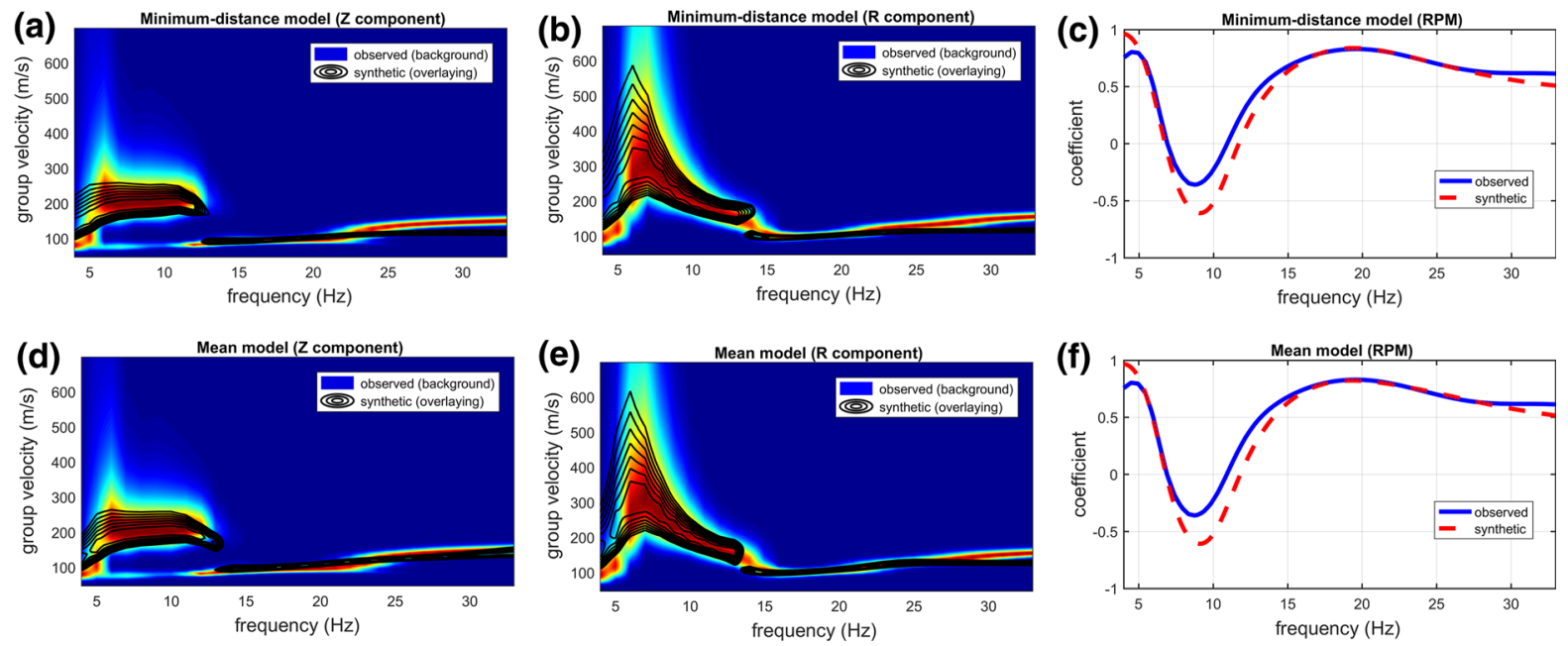

Figure 13

Case study (SO-RPM-HS approach). The two most important models obtained from the joint inversion of the single-offset (48 m) data. Upper plots refer to the minimum-distance model: a vertical-component group-velocity spectra; b radial-component group-velocity spectra; $\mathbf{c}$ RPM frequency curves. Lower plots refer to the mean model (computed by considering all the Pareto optimal models): d vertical-component groupvelocity spectra; e radial-component group-velocity spectra; f RPM frequency curves. For the velocity spectra, the colors in the background represent the field data, while the overlaying black contour lines reflect the synthetic data of the identified models (reported in Fig. 14) 
(MSOR approach). This way we simulated the acquisition of a multi-component MASW dataset with 14 traces (minimum offset and geophone spacing of 3 and $5 \mathrm{~m}$, respectively) (see acquisition parameters in Table 1 and field traces in Fig. 9a). An affordable equipment was then sufficient to record multi-offset and multi-component data suitable for the holistic analyses here considered.

In the following two sections, we present the results obtained from both the multi-offset (MORPM-HS) and single-offset (SO-RPM-HS) approaches.

\subsection{Multi-Offset RPM Holistic (MO-RPM-HS) Analysis}

Figure 9 reports the field traces and the three objects considered for the joint analysis considered in the present paper: the RPM frequency-offset surface and the velocity spectra of the vertical and radial components (since in this case we are dealing with multi-offset data, we here consider the phase velocities).

Two major facts are apparent:

a. the phase velocity spectrum of the $Z$ component shows two distinctive mode jumps at about 8 and $15 \mathrm{~Hz}$ : the signals below $8 \mathrm{~Hz}$ and above $15 \mathrm{~Hz}$ likely pertain to the fundamental mode, while the signal in between relate to higher mode(s);

b. the RPM frequency-offset surface (Fig. 9b) provides evidence of prograde motion in particular between about 5 and $11 \mathrm{~Hz}$.

The radial component (Fig. 9d) appears slightly fuzzy, which might be (at least partly) due to some scattering phenomena induced by old buried utilities (in the area, some old buildings were demolished) that, as shown by Rodríguez-Castellanos et al. (2006), can influence in particular the radial component.

The results of the joint inversion are presented in Figs. 10 and 11. Figure 10 summarizes the agreement between the field and synthetic data of the two most important models (the one having the minimum distance from the utopia point and the mean model computed considering all the Pareto optimal solutions) reported in Fig. 11.
The non-perfect match of the $\mathrm{Z}$ component (Fig. 10a, d) is a clear example of the fact that a joint inversion is a compromise. In fact, in case we would consider the velocity spectrum of just one component (e.g., in this case, the vertical one), we would easily find a model that perfectly matches that spectrum but that is not sufficiently good to match the RPM surface and/or the velocity spectrum of the second component.

In other words, a joint inversion typically produces imperfect matches for the single components, but the obtained subsurface model is more robust with respect to the one that can be obtained from the analysis of one single component only (some details regarding the comparison of the performances of single- and multi-objective inversion will be provided in a work currently in progress).

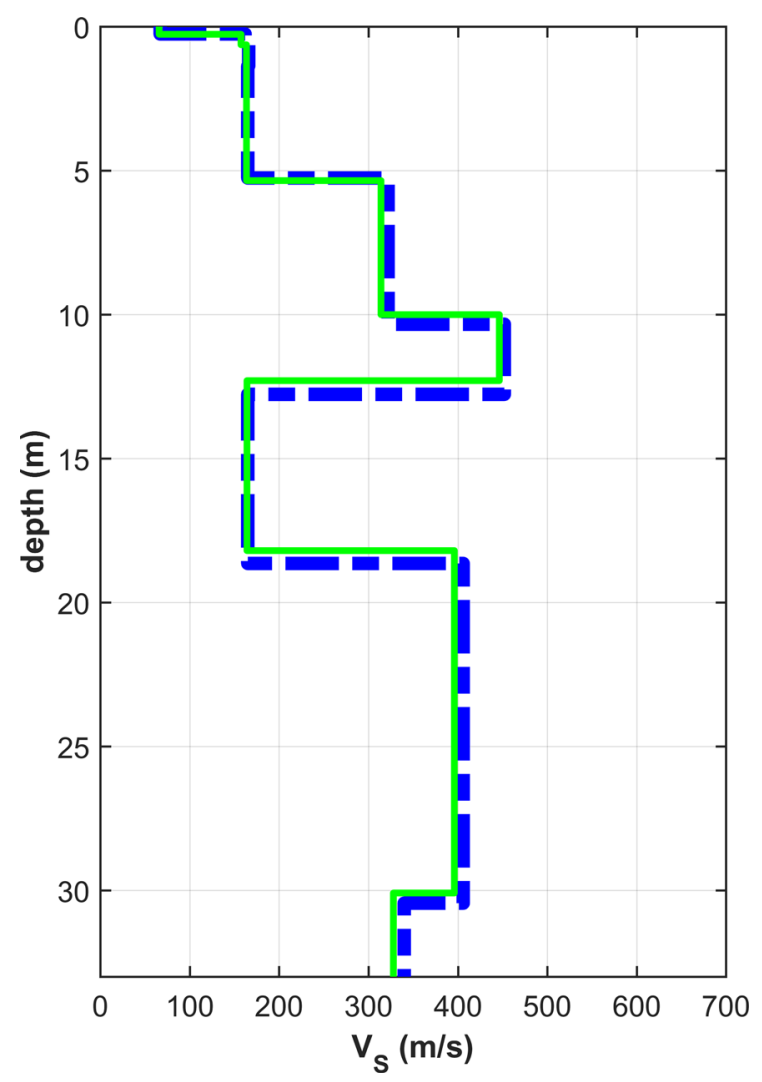

Figure 14

Case study (SO-RPM-HS approach): identified $V_{\mathrm{S}}$ models (shown to approximately two thirds of the considered offset). The dashed blue line indicates the mean model (computed considering all the Pareto optimal models), while the continuous green line shows the minimum-distance model 

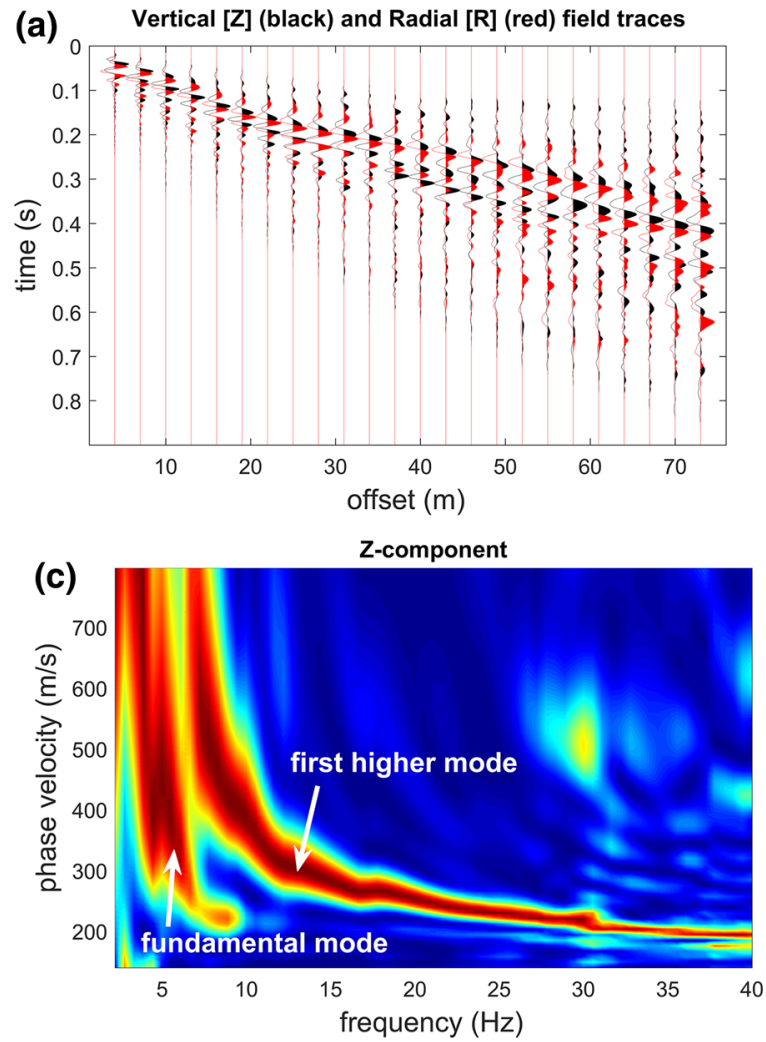
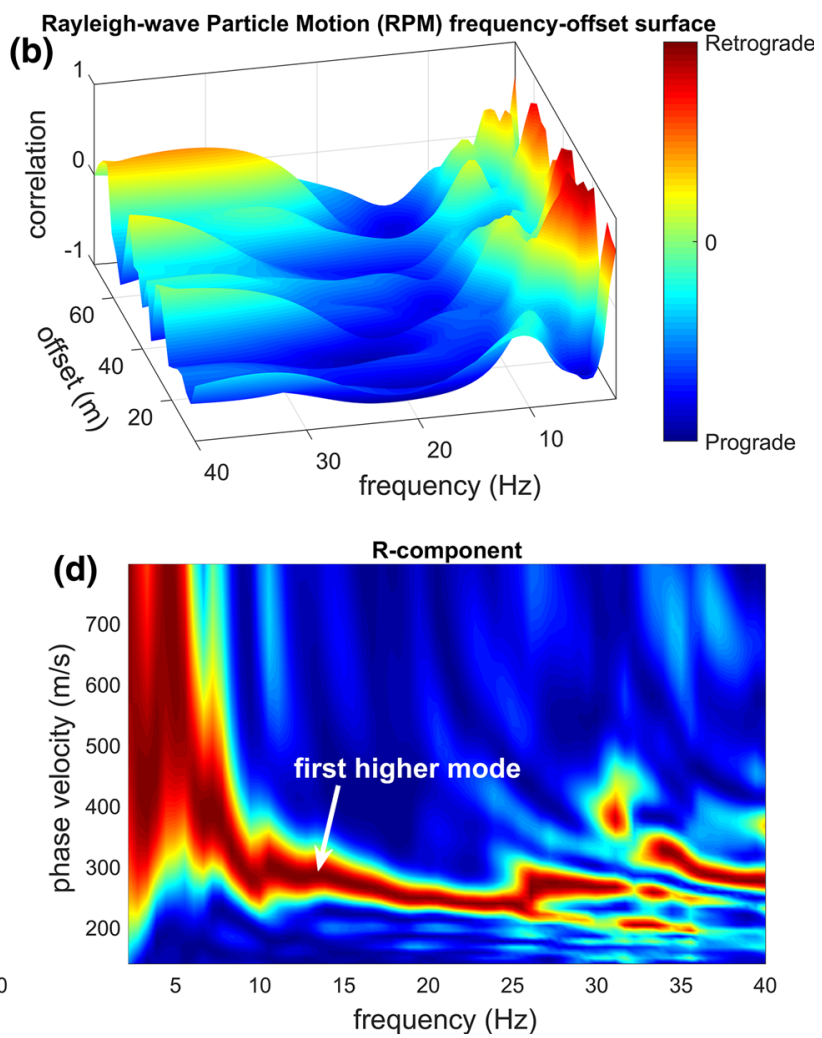

Figure 15

Multi-offset data presented in Dal Moro et al. (2015c) and here considered for the holistic inversion of the Rayleigh waves according to the MO-RPM-HS approach: a seismic traces of the $Z$ and $R$ components; b Rayleigh-wave particle motion (RPM) frequency-offset surface; c phase velocity spectrum of the $Z$ component; $\mathbf{d}$ phase velocity spectrum of the $R$ component. Love waves are presented in Fig. 16
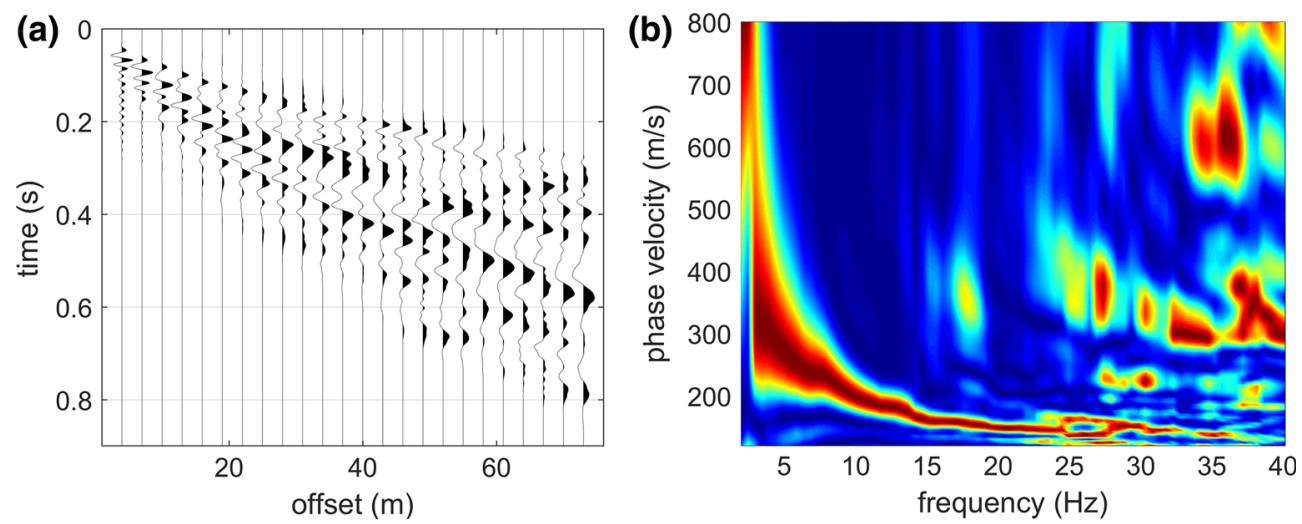

Figure 16

Love waves (transversal component) acquired on the same site as for the data presented in Fig. 15. As highlighted in Dal Moro et al. (2015c), while Rayleigh waves are strongly dominated by the first higher mode, Love waves are apparently largely dominated by the fundamental one 
(a)

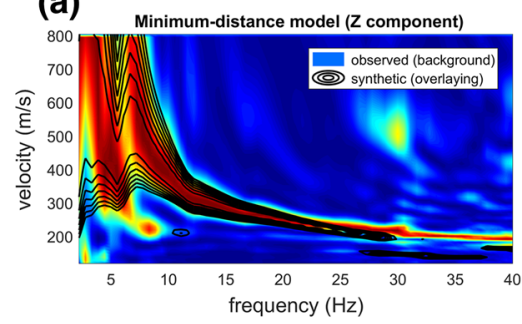

(d)

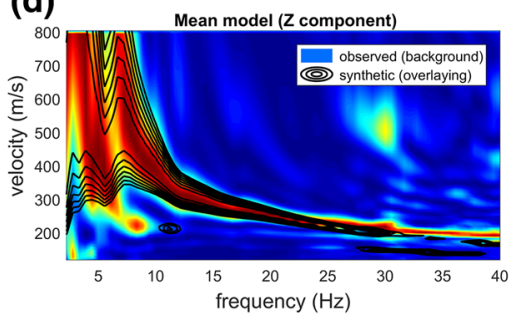

(b)

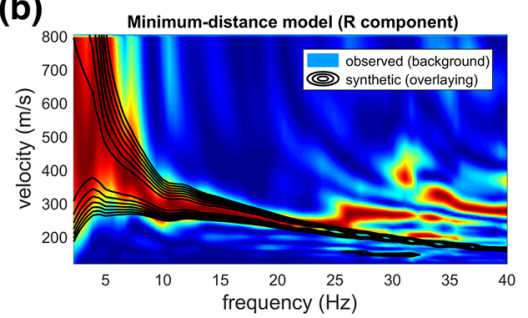

(e)

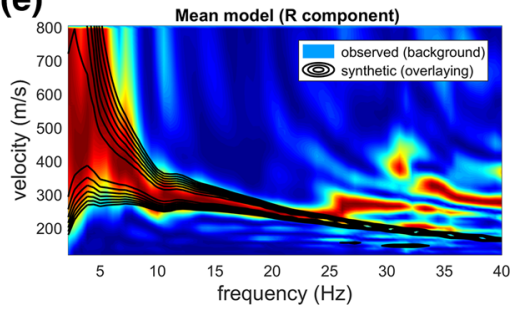

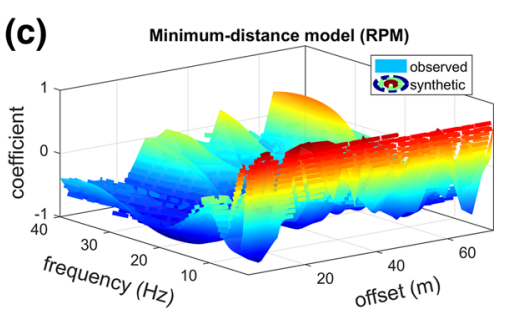

(f)

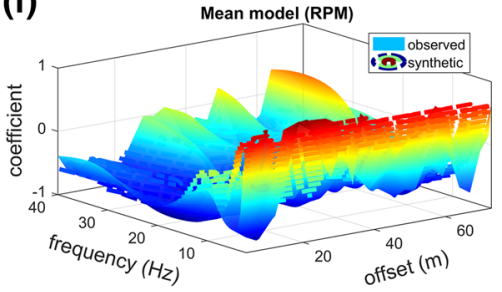

Figure 17

The two most important models obtained from the joint inversion presented in this paper (MO-RPM-HS approach) while considering the dataset presented in Dal Moro et al. (2015c). Upper plots refer to the minimum-distance model: a vertical-component phase velocity spectra; b radial-component phase velocity spectra; c RPM frequency-offset surfaces. Lower plots refer to the mean model (computed by considering all the Pareto front models): d vertical-component phase velocity spectra; e radial-component phase velocity spectra; f RPM frequency-offset surfaces. For the velocity spectra, the colors in the background represent the field data, while the overlying black contour lines reflect the synthetic data of the identified model (reported in Fig. 18a). For the RPM data, the synthetic surface is reported by dashed contour lines with the same color scale as the field data (since the agreement between the field and the synthetic data is extremely good, the two surfaces are visually hardly separable)

From the stratigraphic point of view, the most important feature of the obtained subsurface model is the low-velocity layer (LVL) between about 12 and $18 \mathrm{~m}$, which, considering the overall local geology, can be interpreted as a sandy stratum beneath a gravel layer (gravels dominate the area; see Fig. 8).

\subsection{Single-Offset RPM Holistic (SO-RPM-HS) Analysis}

For the analysis of single-offset data, we considered the 48-m offset traces extracted from the multioffset dataset considered in the previous section (Fig. 12).

The SO-RPM-HS approach varies from the method presented in Dal Moro et al. (2015a, b, 2016), where we considered the combined analysis of the $Z$ and $R$ group-velocity spectra with the RVSR curve (i.e., the ratio between the amplitude spectra of the radial and vertical-components).
The final results of the SO-RPM-HS joint inversion are summarized in Figs. 13 and 14. The overall agreement with the solution obtained from the analysis of multi-offset data (see previous section) is apparent. Although some minor differences are inevitable, the overall velocities and the LVL below the superficial stiffer layers are in fact confirmed (compare Figs. 11 and 14).

\section{Discussion}

The RPM frequency curve (or the frequency-offset surface in case of multi-offset data) represents a quick way for evaluating the tendency of a given site to produce Rayleigh-wave prograde motion which, as pointed out in Trifunac (2009), can represent an additional critical factor for building stability, so that the computation of the RPM curves can provide valuable information also in seismic-hazard assessment studies. 

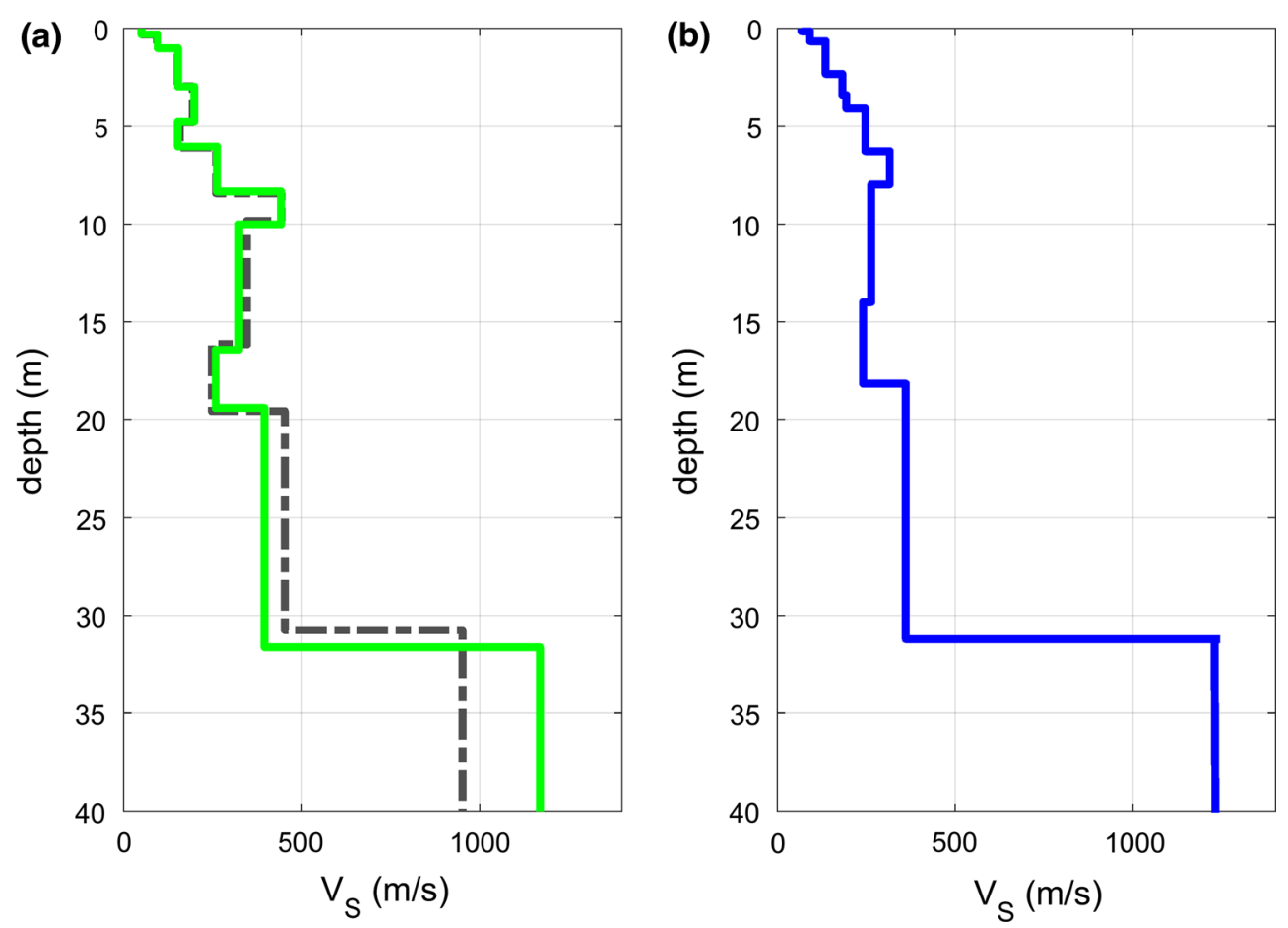

Figure 18

a $V_{\mathrm{S}}$ models of the data presented in Fig. 17. The dashed gray line indicates the mean model (computed considering all the Pareto optimal models), while the continuous green line is the minimum-distance model; $\mathbf{b}$ minimum-distance $V \mathrm{~s}$ model obtained in Dal Moro et al. (2015c) from the joint FVS analysis of the ZVF, RVF and THF components

Because the actual Rayleigh-wave motion depends on the complex interactions of various elements (e.g., high Poisson values, abrupt $V_{\mathrm{S}}$ variations, large-amplitude higher modes, etc.), it is not advisable to simplify the reasons for prograde motion (Dal Moro et al. 2017).

To provide an example of data largely dominated by higher modes and prograde motion, we processed the multi-offset dataset presented in Dal Moro et al. (2015c) according to the procedure considered in this study.

While in Dal Moro et al. (2015c) we considered the multi-component joint inversion of the phase velocity spectra of Love and Rayleigh (both radial and vertical components) waves, we here focus on Rayleigh waves only and consider the joint analysis of the phase velocity spectra (vertical and radial components) together with the RPM frequency-offset surface.

The field traces of the $Z$ and $R$ components are shown in Fig. 15a and the RPM frequency-offset surface in Fig. 15b. In the 5-40 Hz frequency range, Rayleigh-wave motion is apparently strongly prograde (for all the offsets), while at lower frequencies the motion is retrograde.

If we consider the $\mathrm{Z}$ and $\mathrm{R}$ phase velocity spectra (Fig. 15c, d), it is quite remarkable how both the components are strongly dominated by higher modes (further details in Dal Moro et al. 2015c).

Actually, in this case, the highly prograde motion observed for frequencies higher than about $5 \mathrm{~Hz}$ (Fig. 15b), is likely related to the large-amplitude of the first higher mode that dominate Rayleigh waves at those frequencies.

On the other hand, as often observed (Safani et al. 2005; Dal Moro and Ferigo 2011; Xia et al. 2012; Dal Moro 2014), Love waves are clearly dominated by the fundamental mode (Fig. 16).

The results of the MO-RPM-HS joint inversion presented in Figs. 17 and 18a appear in good agreement with the solution presented in Dal Moro et al. (2015c) and reported in Fig. 18b, which largely relied 

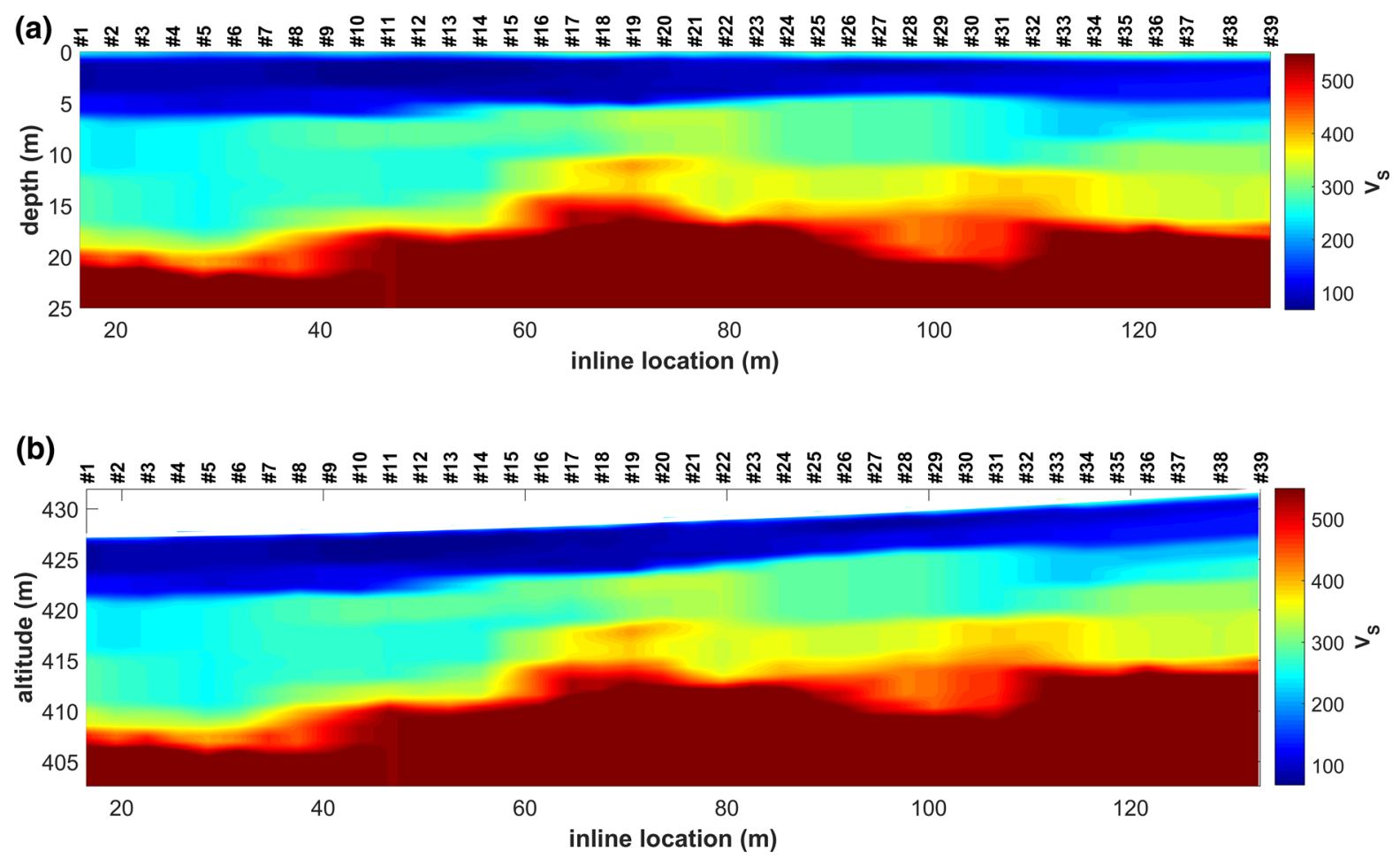

Figure 19

Example of a 2D Vs section obtained from the joint analysis described in this paper: a $V \mathrm{~s}$ section as a function of the inline position and depth from the surface; $\mathbf{b} V$ s section as a function of the inline position and altitude (above sea level). Labels reported at the top of the two sections indicate the shot number. The details regarding two of the 39 shots are reported in Figs. 20 and 21

on the Love waves (that are dominated by the fundamental mode and, consequently, are less prone to modal dispersion curve misinterpretations).

Actually, since it is based on the holistic analysis of Rayleigh waves only, the proposed approach can be particularly relevant for the unambiguous exploration of large areas, without the need of the joint acquisition of Love waves, which, when dealing with several hundreds or thousands of shots, can become quite problematic (see introductory paragraph).

Figure 19 reports the $2 \mathrm{D} V_{\mathrm{S}}$ section obtained by analyzing a northern Switzerland dataset according to the MO-RPM-HS approach. The datasets consists of 39 multi-component $(Z+R)$ and multi-offset shots acquired following a classical roll-along procedure (average shot spacing equal to $3 \mathrm{~m}$ ).

Each shot was analyzed according to the presented methodology and the $39 V_{\mathrm{S}}$ profiles were then used to create the presented $2 \mathrm{D}$ section.
The details regarding the analysis of two of the 39 shots are presented in Figs. 20 and 21. It is possible to clearly delineate a relatively stiff, superficial, and thin cover (filling materials) over a soft lacustrine sequence overlaying a very hard till/gravel sequence typical of the investigated area.

\section{Conclusion}

In the present paper, we introduced and discussed the joint analysis of the RPM data together with the velocity spectra of the vertical and radial components of Rayleigh waves.

Such a method can be applied both to multi-offset and single-offset (multi-component) data. In case of multi-offset data, we refer to the approach as MORPM-HS, while in case of single-offset data we use the expression SO-RPM-HS. 
(a)

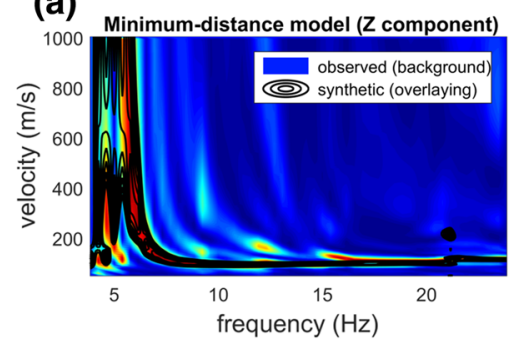

(d)

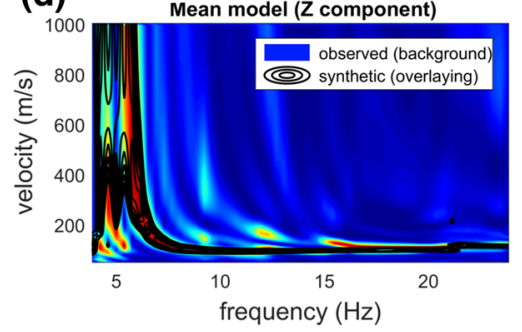

(b)

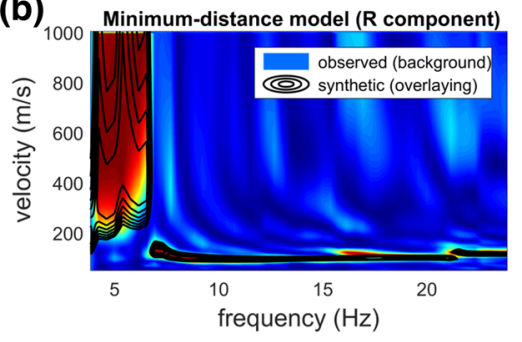

(e)

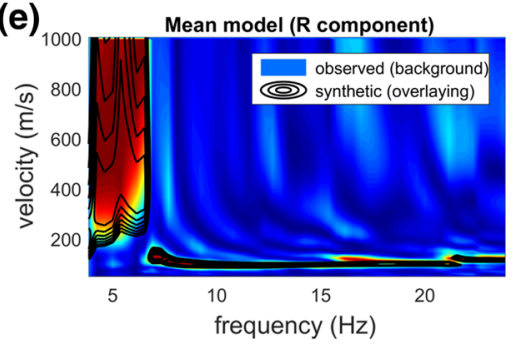

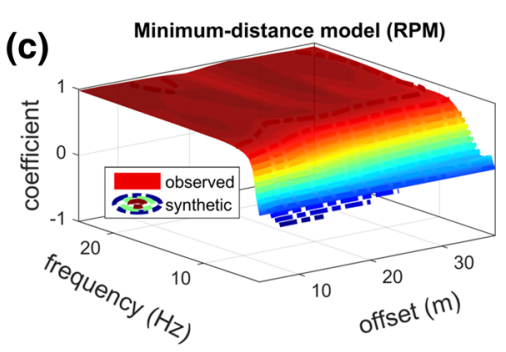

(f)

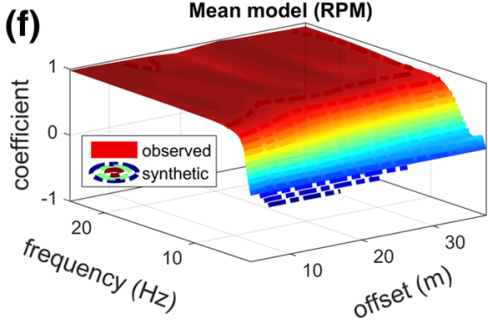

\section{shot\#6}

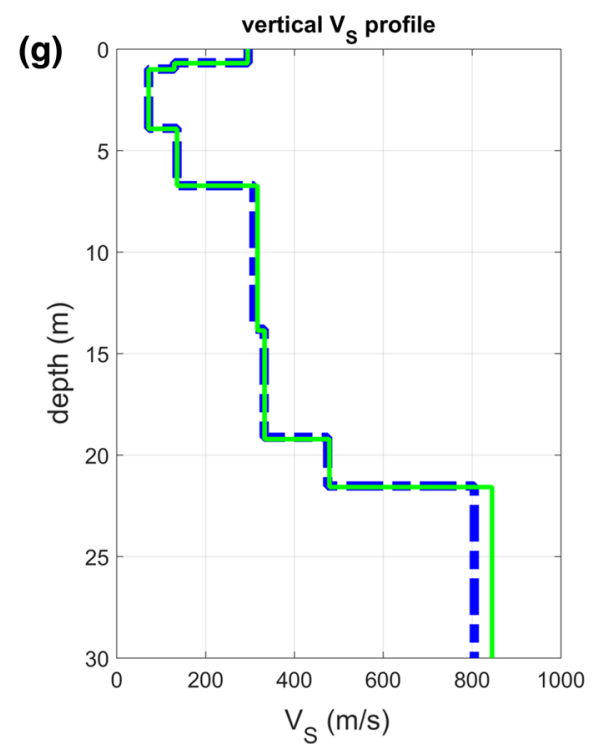

Figure 20

Shot \#6 (see shot location in Fig. 19): joint inversion accomplished according to the MO-RPM-HS approach. Upper plots refer to the minimum-distance model: a vertical-component phase velocity spectra; b radial-component phase velocity spectra; $\mathbf{c}$ RPM frequency-offset surfaces. Lower plots refer to the mean model (computed by considering all the Pareto front models): d vertical-component phase velocity spectra; e radial-component phase velocity spectra; f RPM frequency-offset surfaces. The two $V$ s profiles are reported in the $\mathbf{g}$ plot. For the velocity spectra, the colors in the background represent the field data, while the overlaying black contour lines reflect the synthetic data of the identified models. For the RPM data, the synthetic surface is reported by dashed contour lines with the same color scale as the field data (since the agreement between the field and synthetic data is extremely good, the two surfaces are visually hardly separable)

While the velocity spectra describe the velocities along the two $Z$ and $R$ axes (the two velocity spectra are in general different, so provide complementary information), the RPM data characterize the actual particle motion induced by the Rayleigh-wave propagation. Such particle motion is a function of both frequency and offset and is generally often far from simple retrograde motion, as often simplistically assumed. 
(a)

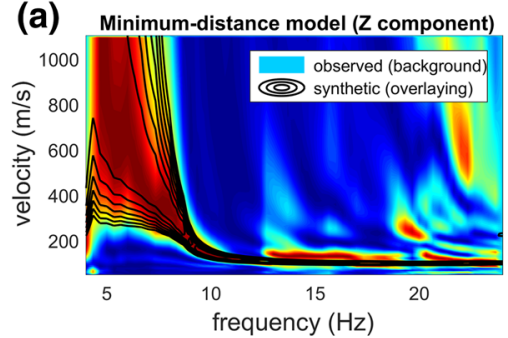

(d)

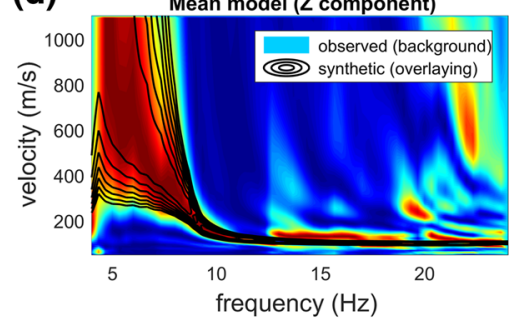

(b)

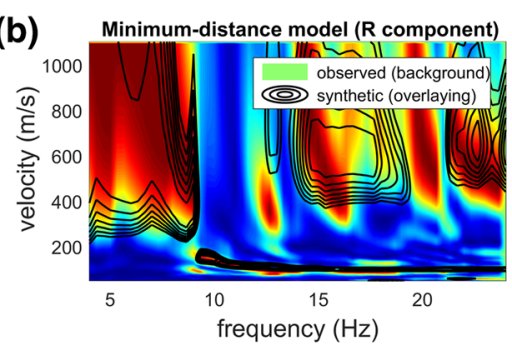

(e)

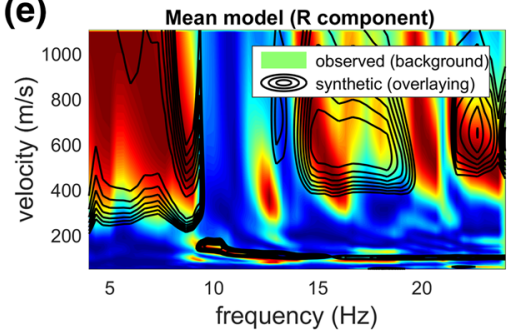

(c) Minimum-distance model (RPM)

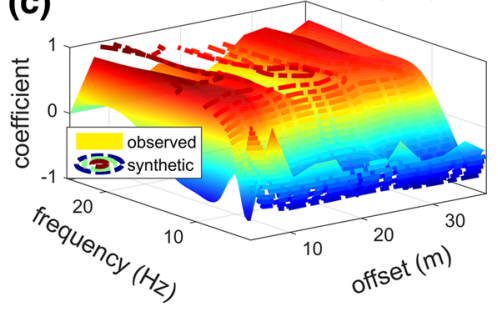

(f)

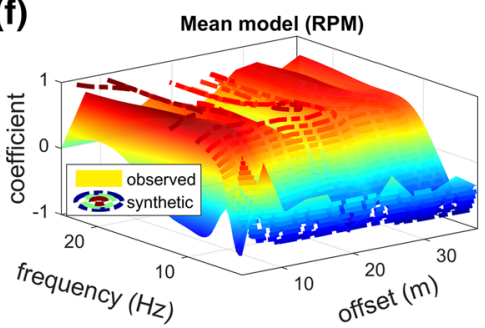

shot\#22

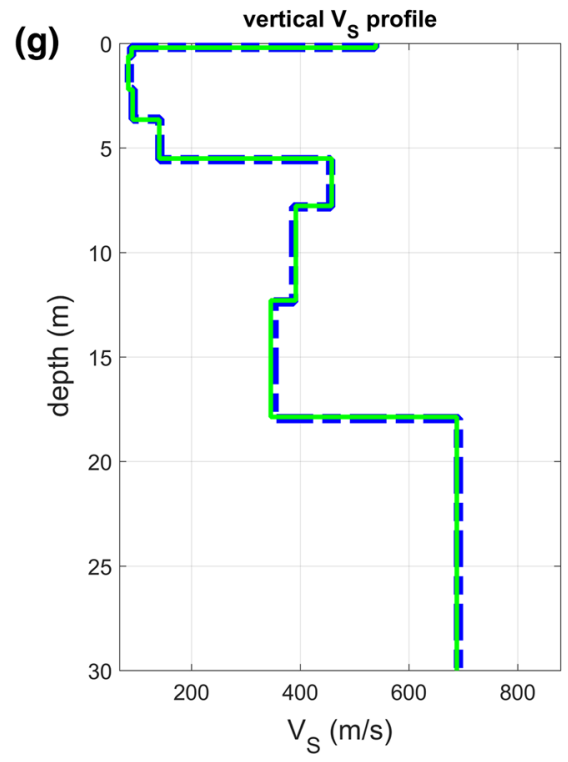

Figure 21

Shot \#22 (see shot location in Fig. 19): joint inversion accomplished according to the MO-RPM-HS approach. Upper plots refer to the minimum-distance model: a vertical-component phase velocity spectra; b radial-component phase velocity spectra; $\mathbf{c}$ RPM frequency-offset surfaces. Lower plots refer to the mean model (computed by considering all the Pareto front models): d vertical-component phase velocity spectra; e radial-component phase velocity spectra; f RPM frequency-offset surfaces. The Vs two profiles are reported in the $\mathbf{g}$ plot. For the velocity spectra, the colors in the background represent the field data, while the overlaying black contour lines reflect the synthetic data of the identified models. For the RPM data, the synthetic surface is reported by dashed contour lines with the same color scale as the field data (since the agreement between the field and synthetic data is extremely good, the two surfaces are visually hardly separable)

Unlike the polarity analysis proposed by Gribler et al. (2016) and the RVSR curve used in Dal Moro et al. (2015a, b, 2016), the RPM frequency-offset data can be determined even when using vertical and horizontal geophones with different response curves.
Furthermore, compared with the approach adopted in Gribler et al. (2016), the RPM curve/surface describes the Rayleigh-wave motion more accurately because the correlation coefficients are computed as a function of the frequency (and offset). 
Being based on Rayleigh waves only, the presented approach can be significantly efficient in particular for the unambiguous exploration of large areas.

In this case, in fact, the joint acquisition of both Rayleigh and Love waves could be problematic during the acquisition of hundreds or thousands of shots.

It must be emphasized that the dispersion analysis of a single component (typically represented by the vertical component of Rayleigh waves) is often insufficient to solve the ambiguities related to the interpretation of the velocity spectra and the nonuniqueness of the solution.

We must also consider that passive seismics does not represent an economically feasible approach because the recording times are too long when exploring large areas for geotechnical purposes.

The approach presented in the present paper represents a possible solution that allows the joint analysis of three "objects" and the implementation of a well-constrained inversion that can provide a robust subsurface model.

It is also important to underline that the polarities of the geophones used to acquire the data must be known and that the computation of the synthetic traces must be clearly consistent.

In other words, the convention adopted by the sensors must be known (e.g., about the vertical geophones, it must be known if the plus sign refers to an upward or downward motion; for a wide discussion about these issues see Brown et al. 2002).

From a very practical point of view, it is also important to check the polarity of all the geophones because, in our experience, it is not uncommon that, in a set of 24 geophones, one or two geophones have opposite polarities.

\section{Acknowledgements}

This work was supported by the Deanship of Scientific Research of the King Saud University (Riyadh, Saudi Arabia - PRG-1436-06 Research Grant) and by the Institute of Rock Structure and Mechanics (Czech Academy of Sciences - RVO 67985891 Research Grant). The authors would like to express his gratitude to Lorenz Keller (roXplore,
Switzerland) for the permission to publish the data presented in the Discussion.

Open Access This article is distributed under the terms of the Creative Commons Attribution 4.0 International License (http:// creativecommons.org/licenses/by/4.0/), which permits unrestricted use, distribution, and reproduction in any medium, provided you give appropriate credit to the original author(s) and the source, provide a link to the Creative Commons license, and indicate if changes were made.

\section{REFERENCES}

Boxberger, T., Picozzi, M., \& Parolai, S. (2011). Shallow geology characterization using Rayleigh and love wave dispersion curves derived by seismic noise array measurements. Journal of Applied Geophysics, 75, 345-354.

Brown, R. J., Stewart, R. R., \& Lawton, D. C. (2002). A proposed polarity standard for multicomponent seismic data. Geophysics, 67, 1028-1037.

Carcione, J. M. (1992). Modeling anelastic singular Surface waves in the Earth. Geophysics, 57, 781-792.

Dal Moro, G. (2014). Surface wave analysis for near surface applications, Elsevier, Amsterdam, The Netherlands, p. 252, ISBN 9780128007709.

Dal Moro, G., Al-Arifi, N., \& Moustafa, S. R. (2017). Analysis of Rayleigh-wave particle motion from active seismics. Bulletin of the Seismological Society of America, 107, 51-62.

Dal Moro, G., Coviello, V., \& Del Carlo, G. (2014). Shear-wave velocity reconstruction via unconventional joint analysis of seismic data: A case study in the light of some theoretical aspects, IAEG XII CONGRESS - Turin, September 15-19, 2014. In G. Lollino, A. Manconi, F. Guzzetti, M. Culshaw, P. Bobrowsky, F. Luino (Eds.), Engineering geology for society and territoryVolume 5 (pp. 1177-1182). Springer International Publishing.

Dal Moro, G., \& Ferigo, F. (2011). Joint analysis of Rayleigh and love wave dispersion for near-surface studies: issues, criteria and improvements. Journal of Applied Geophysics, 75, 573-589.

Dal Moro, G. \& Keller, L. (2015). Optimizing the exploration of vast areas via multi-component surface-wave analysis. Proceedings EAGE 2015, June 1-5 2015 (Madrid, Spain), Extended Abstract.

Dal Moro, G., Keller, L., Moustafa, S. R., \& Al-Arifi, N. (2016). Shear-wave velocity profiling according to three alternative approaches: a comparative case study. Journal of Applied Geophysics, 134, 112-124.

Dal Moro, G., Keller, L., \& Poggi, V. (2015a). A comprehensive seismic characterization via multi-component analysis of active and passive data. First Break, 33, 45-53.

Dal Moro, G., Moura, R. M., \& Moustafa, S. R. (2015b). Multicomponent joint analysis of surface waves. Journal of Applied Geophysics, 119, 128-138.

Dal Moro, G., Ponta, R., \& Mauro, R. (2015c). Unconventional optimized surface wave acquisition and analysis: comparative tests in a perilagoon area. Journal of Applied Geophysics, 114, 158-167.

Dal Moro, G., \& Pipan, M. (2007). Joint inversion of surface wave dispersion curves and reflection travel times via multi-objective 
evolutionary algorithms. Journal of Applied Geophysics, 61, 56-81.

Dal Moro, G., Pipan, M., Forte E., \& Finetti, I. (2003). Determination of Rayleigh wave dispersion curves for near surface applications in unconsolidated sediments, Proceedings SEG, 73th Annual Int. Mtg, Dallas, Texas, Oct 2003.

Dziewonsky, A., Bloch, S., \& Landisman, N. (1969). A technique for the analysis of transient seismic signals. Bulletin of the Seismological Society of America, 59, 427-444.

Foti, S., Lai, C.G., Rix, G.J., \& Strobbia, C. (2014). Surface wave methods for near-surface site characterization. CRC Press.

Gribler, G., Liberty, L. M., Mikesell, T. D., \& Michaels, P. (2016). Isolating retrograde and prograde Rayleigh-wave modes using a polarity mute. Geophysics, 81, V379-V385.

Herrmann, R. B. (2013). Computer programs in seismology: an evolving tool for instruction and research. Seismological Research Letters, 84, 1081-1088.

Hobiger, M., Bard, P.-Y., Cornou, C., \& Le Bihan, N. (2009). Single station determination of Rayleigh wave ellipticity by using the random decrement technique (RayDec). Geophysical Research Letters, 36, L14303.

Luo, Y., Xia, J., Xu, Y., \& Zeng, C. (2011). Analysis of groupvelocity dispersion of high-frequency Rayleigh waves for nearsurface applications. Journal of Applied Geophysics, 74, 157-165.

Malischewsky, P. G., Scherbaum, F., Lomnitz, C., Tuan, T. T., Wuttke, F., \& Shamir, G. (2008). The domain of existence of prograde Rayleigh wave particle motion for simple models. Wave Motion, 45, 556-564.

O'Connell, D. R. H., \& Turner, J. P. (2011). Interferometric multichannel analysis of surface waves (IMASW). Bulletin of the Seismological Society of America, 101, 2122-2141.

Poggi, V., \& Fäh, D. (2010). Estimating Rayleigh wave particle motion from three-component array analysis of ambient vibrations. Geophysical Journal International, 180, 251-267.

Rodríguez-Castellanos, A., Sánchez-Sesma, F. J., Luzón, F., \& Martin, R. (2006). Multiple scattering of elastic waves by subsurface fractures and cavities. Bulletin of the Seismological Society of America, 96, 1359-1374.

Ryden, N., Park, C.B., Ulriksen, P., \& Miller R.D. (2003). Lamb wave analysis for nondestructive testing of concrete plate structures. Proceedings of the Symposium on the Application of Geophysics to Engineering and Environmental Problems (SAGEEP 2003), San Antonio, TX, April 6-10, INF03.

Safani, J., O’Neill, A., Matsuoka, T., \& Sanada, Y. (2005). Applications of Love wave dispersion for improved Shear-wave velocity imaging. Journal of Environmental and Engineering Geophysics, 10, 135-150.

Sawaragi, Y., Nakayama, H., \& Tamino, T. (1985). Theory of multiobjective optimization (p. 296). Orlando: Academic Press.

Tanimoto, T., \& Rivera, L. (2005). Prograde Rayleigh wave motion. Geophysical Journal International, 162, 399-405.

Trifunac, M. D. (2009). The role of strong motion rotations in the response of structures near earthquake faults. Soil Dynamics and Earthquake Engineering, 29, 382-393.

Van Veldhuizen, D.A. \& Lamont, G.B. (1998). Evolutionary Computation and Convergence to a Pareto Front. In: Koza, John R. (Ed.), Late Breaking Papers at the Genetic Programming 1998 Conference. Stanford University, pp. 221-228.

Xia, J., Miller, R. D., \& Park, C. B. (1999). Estimation of nearsurface Shear-wave velocity by inversion of Rayleigh waves. Geophysics, 64, 691-700.

Xia, J., Xu, Y., Luo, Y., Miller, R. D., Cakir, R., \& Zeng, C. (2012). Advantages of using multichannel analysis of love waves (MALW) to estimate near-surface shear-wave velocity. Surveys In Geophysics, 33, 841-860.

Zhang, S. X., \& Chan, L. S. (2003). Possible effects of misidentified mode number on Rayleigh wave inversion. Journal of Applied Geophysics, 53, 17-29.

Zhang, C., Liu, Q., \& Deng, P. (2017). Surface motion of a half-space with a semicylindrical canyon under P, SV, and Rayleigh waves. Bulletin of the Seismological Society of America, 107, 809-820.

Zitzler, E., \& Thiele, L. (1999). Multiobjective evolutionary algorithms: a comparative case study and the strength Pareto approach. IEEE Transactions on Evolutionary Computation, 3, 257-271. 